\title{
What Can We Learn From a Biomimetic Model of Nature's Oxygen-Evolving Complex?
}

\author{
Satadal Paul, ${ }^{,}$Nicholas Cox, ${ }^{a b}$ Dimitrios A. Pantazis ${ }^{* a}$
}

a) Max Planck Institute for Chemical Energy Conversion,

Stiftstr. 34-36, 45470 Mülheim an der Ruhr, Germany.

b) Research School of Chemistry, Australian National University, Canberra, ACT 2601, Australia.

dimitrios.pantazis@cec.mpg.de 
ABSTRACT: A recently reported synthetic complex with a $\mathrm{Mn}_{4} \mathrm{CaO}_{4}$ core represents a remarkable structural mimic of the $\mathrm{Mn}_{4} \mathrm{CaO}_{5}$ cluster in the oxygen-evolving complex (OEC) of photosystem II (Zhang et al., Science 2015, 348, 690). Oxidized samples of the complex show EPR signals at $g \approx 4.9$ and $g \approx 2$, similar to those associated with the OEC in its $\mathrm{S}_{2}$ state $(g \approx 4.1$ from an $S=5 / 2$ form and $g \approx 2$ from an $S=1 / 2$ form), suggesting similarities in electronic as well as in geometric structure. We use quantum chemical methods to characterize the synthetic complex in various oxidation states, to compute its magnetic and spectroscopic properties, and to establish connections with reported data. Only one energetically accessible form is found for the oxidized " $\mathrm{S}_{2}$ state" of the complex. It has a ground spin state of $S=5 / 2$ and EPR simulations confirm it can be assigned to the $g \approx 4.9$ signal. However, no valence isomer with an $S=1 / 2$ ground state is energetically accessible, a conclusion supported by a wide range of methods, including density matrix renormalization group (DMRG) with full valence active space. Alternative candidates for the $g \approx 2$ signal were explored, but no low-spin/low-energy structure was identified. Therefore, our results suggest that despite geometric similarities the synthetic model does not mimic the valence isomerism that is the hallmark of the OEC in its $\mathrm{S}_{2}$ state, most probably because it lacks a coordinatively flexible oxo bridge. Only one of the observed EPR signals can be explained by a structurally intact high-spin one-electron oxidized form, while the other originates from an as yet unidentified rearrangement product. Nevertheless, this model provides valuable information for understanding the high-spin EPR signals of both the $S_{1}$ and $S_{2}$ states of the OEC in terms of coordination number and Jahn-Teller axis orientation of the Mn ions, with important consequences for the development of magnetic spectroscopic probes to study S-state intermediates immediately prior to $\mathrm{O}-\mathrm{O}$ bond formation. 


\section{INTRODUCTION}

Light-driven water oxidation in the photosystem II (PSII) of oxygenic photosynthetic organisms is the process that ultimately sustains most life on our planet. ${ }^{1-2}$ The catalytic site of PSII responsible for water oxidation, ${ }^{3-5}$ known as the oxygen-evolving complex (OEC), harbors a cluster composed of four $\mathrm{Mn}$ and one $\mathrm{Ca}$ ions bridged by five oxo groups $\left(\mathrm{Mn}_{4} \mathrm{CaO}_{5}\right)$ and supported by carboxylate amino acid side chains. ${ }^{6-8}$ As described by Joliot and Kok, ${ }^{9}$ the catalytic cycle of the OEC involves five oxidation states $\mathrm{S}_{i}$ where $i=0-4$ denotes the oxidation level of the cluster. The $\mathrm{S}_{0}$ state is the most reduced physiological form, $\mathrm{Mn}(\mathrm{III})_{3} \mathrm{Mn}(\mathrm{IV})$, advancing to a $\mathrm{Mn}(\mathrm{IV})_{4} \mathrm{~S}_{3}$ state ${ }^{10-13}$ before the final oxidation step to the transient $\mathrm{S}_{4}$ state that evolves dioxygen and resets the system to $\mathrm{S}_{0} \cdot{ }^{14}$ Owing to its extraordinary ability to catalyze this challenging redox transformation efficiently, the OEC is considered a blueprint for the development of synthetic water-oxidizing systems based on first-row transition metals. ${ }^{15-22}$

Synthetic chemistry has targeted various structural aspects of the OEC, including nuclearity, metal composition, and bonding topology. ${ }^{23-26}$ While there is still no synthetic model of the OEC that precisely reproduces the stoichiometry and bonding topology of the inorganic core, significant advances have been made in recent years. Among the most recent ones, the quest for close structural mimics of the OEC has led to a class of high-valent $\mathrm{Mn}_{3} \mathrm{CaO}_{4}$ cubanes. ${ }^{27-29}$ The core of these complexes models part of the OEC in one of the $S_{2}$ state conformers (the $S=5 / 2$ "closed-cubane" form of the $S_{2}$ state, see below). These complexes have been extensively studied for their properties and have served as a platform to examine specific chemical modifications, such as the effect of the redox-inactive cations on the reduction potential. ${ }^{30-34} \mathrm{~A}$ more recent significant advance was achieved by Zhang et al., ${ }^{35}$ who reported the synthesis of $\left(\left[\mathrm{Mn}_{4} \mathrm{CaO}_{4}\left(\mathrm{BuCO}_{2}\right)_{8}\left(\mathrm{BuCO}_{2} \mathrm{H}\right)_{2}(\right.\right.$ py) $](\mathrm{Bu}$ : tert-butyl; py: pyridine), shown as complex 1 in Figure 
1, that contains a fourth $\mathrm{Mn}$ ion attached to one of the oxygen bridges of a $\mathrm{Mn}_{3} \mathrm{CaO}_{4}$ cubane. The core of this complex is similar to the above-mentioned $\mathrm{Mn}_{3} \mathrm{CaO}_{4}$ cubanes with the addition of an extra Mn ion that resembles the "dangler" Mn4 of the OEC. It is important to note that the structural analogy is not exact: the stoichiometry and bonding topology of the core are different because complex 1 lacks an O bridge between Mn3 and Mn4 compared to the OEC and hence the "dangler" $\mathrm{Mn}$ ion is not a part of a bis- $\mu$-oxo $\mathrm{Mn}_{2} \mathrm{O}_{2}$ subunit as it is in the OEC. The absence of this bridge (O4 in the $\mathrm{OEC}$ core) implies that the existing $\mathrm{O} 4$ bridge (which corresponds topologically to the $\mathrm{O} 5$ of the $\mathrm{OEC}$ ) is chemically distinct from the natural system. In the OEC, it is present as a semi-flexible bridge ${ }^{36-38}$ that connects $\mathrm{Ca}$ and two $\mathrm{Mn}$ ions depending on the isomeric form (either Mn1 and Mn3 or Mn3 and Mn4) but it does not formally function as a $\mu_{4-}$ oxo bridge like in the more compact core of complex 1.
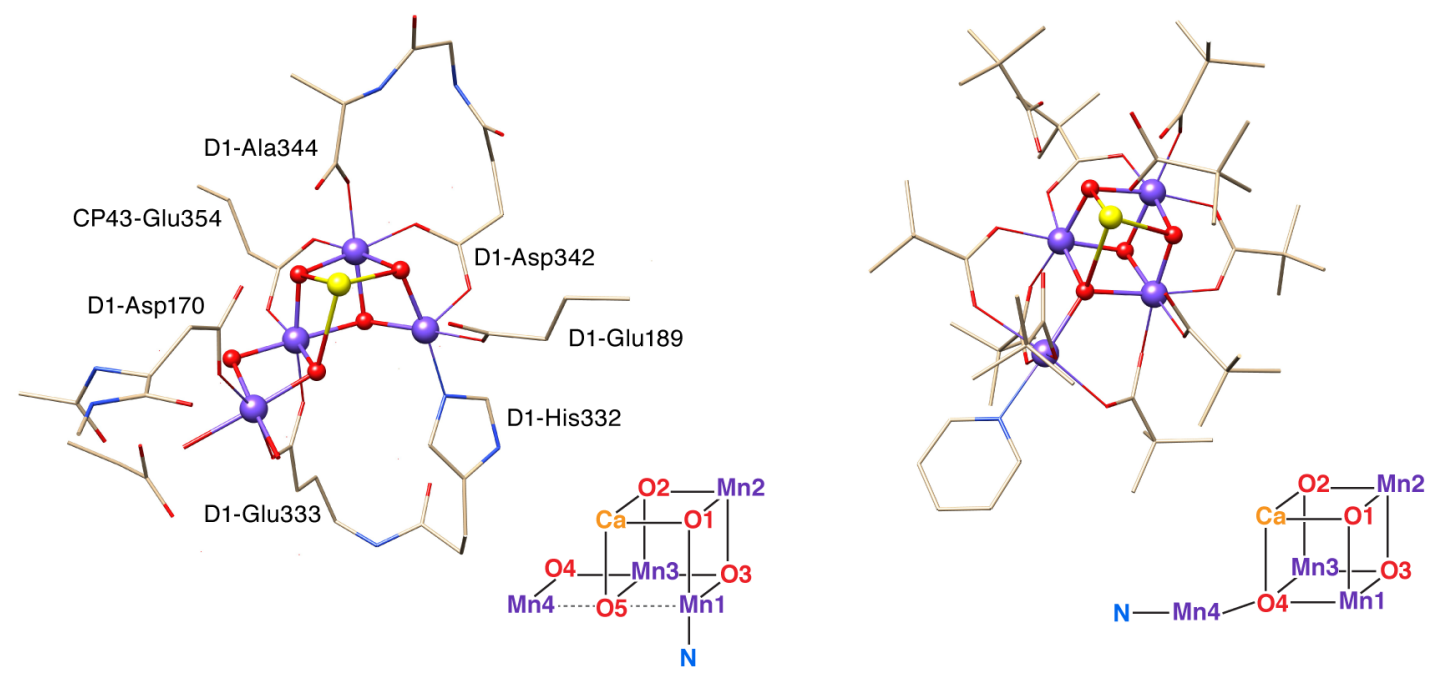

Figure 1. The oxygen-evolving complex of photosystem II (left) and the synthetic complex 1 (right).

On the basis of bond-valence sum analysis, ${ }^{39-40}$ the Mn oxidation states of complex 1 were assigned as $\mathrm{Mn}(\mathrm{III})_{2} \mathrm{Mn}(\mathrm{IV})_{2},{ }^{35}$ precisely the same as the $\mathrm{S}_{1}$ state of the OEC. In addition to structural similarity, the synthetic model resembles the natural system in its ability to accumulate 
oxidizing equivalents similar to the transitions between the $\mathrm{S}_{0}-\mathrm{S}_{4}$ states of the OEC, while a most striking aspect is the simultaneous appearance of two EPR signals in its one-electron oxidized form ("S $\mathrm{S}_{2}$ state"), at $g \approx 4.9$ and $g \approx 2 .{ }^{35}$ These signals are reminiscent of the $\mathrm{S}_{2}$ state of the biological cluster with metal oxidation states $\mathrm{Mn}(\mathrm{III}) \mathrm{Mn}(\mathrm{IV})_{3}$, where two EPR signals at $g \geq 4.1$ and $g \approx 2$ can be observed. ${ }^{41-46}$ These arise from two valence isomers that differ in the connectivity of the $\mathrm{O} 5$ bridge. ${ }^{36-38}$ A "closed-cubane" form with the unique $\mathrm{Mn}(\mathrm{III})$ at the Mn4 site has a spin $S=5 / 2$ and is associated with the $g \geq 4.1$ signal, while an "open-cubane" form with the $\mathrm{Mn}(\mathrm{III})$ at $\mathrm{Mn} 1$ gives rise to the $S=1 / 2, g \approx 2$ signal (Figure 2 ). ${ }^{36}$ This special type of structural flexibility has been suggested to be crucial in controlling the spin state and reactivity of the cluster at the late catalytic steps, ${ }^{47}$ in gating the complex $\mathrm{S}_{2}-\mathrm{S}_{3}$ transition, ${ }^{48-49}$ and in controlling water coordination in the $\mathrm{S}_{3}$ state..$^{48,50-51}$ This then leads to the question as to whether the EPR signals reported for the oxidized form of complex $\mathbf{1}$ could have a similar structural explanation?
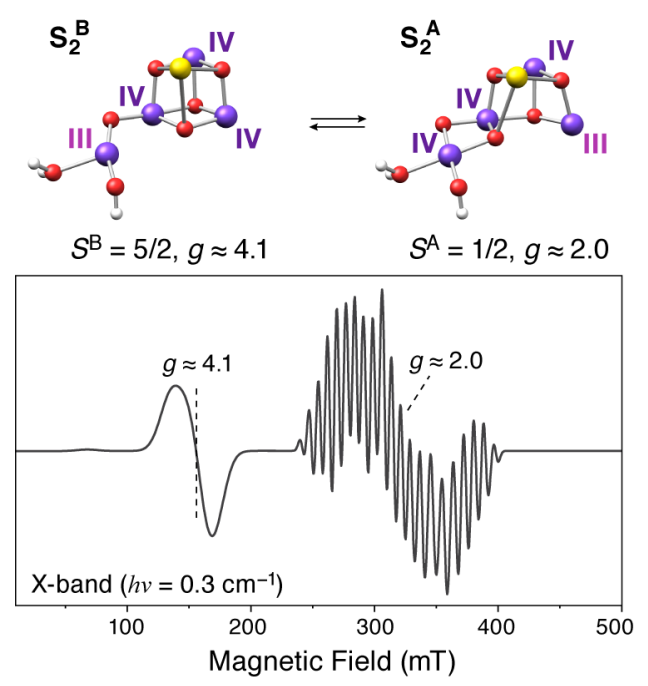

Figure 2. The two interconvertible structural forms in the $S_{2}$ state of the OEC that correspond to the two EPR signals observed for this state (simulated spectrum adapted from Ref. ${ }^{36}$ ). 
The unprecedented combination of structural and spectroscopic parallels between complex $\mathbf{1}$ and the OEC inspired us to investigate the properties of the synthetic complex using spectroscopy-oriented quantum chemistry. We analyze the geometry, electronic structure, magnetic properties and spectroscopic signatures of complex $\mathbf{1}$ in its neutral, cationic and anionic states, some of which correspond to catalytic states of the OEC. Particular emphasis is placed on the "S $\mathrm{S}_{2}$ state" of complex 1 because of its unexpected spectroscopic signature. All possible valence isomers, as well as a large number of structurally rearranged models of complex $\mathbf{1}$ are examined to establish possible mappings between structural parameters, spin properties and the EPR spectra. The results suggest that the apparent similarities in spectroscopic behavior between the synthetic and the biological system do not have a common structural origin.

\section{METHODOLOGY}

The crystallographic coordinates of complex $\mathbf{1}$ were used as the starting geometry, with addition of missing hydrogen atoms. All calculations used the ORCA package.52 Full optimizations were performed in the high spin (HS) and, for selected cases, in the lowest-energy broken-symmetry (BS) states, with the TPSSh functional..$^{53}$ TPSSh has been shown to perform well for a range of systems and properties ${ }^{5455}$ including manganese-oxo complexes and the OEC..12, ${ }^{32,36,5660}$ Scalar relativistic effects were included through the zeroth-order regular approximation (ZORA), ${ }^{61-63}$ retaining one-center terms and using ZORA-recontracted ${ }^{6+65}$ def2-TZVP basis sets ${ }^{66}$ for

all elements except $\mathrm{C}$ and $\mathrm{H}$, for which ZORA-SVP basis sets were used. The resolution of identity (RI) approximation to Coulomb exchange with a decontracted def2-TZVP/J auxiliary basis set $^{67}$ and the chain-of-spheres approximation to exact exchange were employed. ${ }^{8}$ All calculations used the dispersion corrections proposed by Grimme, with Becke-Johnson damping 
(D3BJ) ${ }^{6970}$ The conductor-like screening model (COSMO) $)^{n 1}$ with a dielectric constant of $\varepsilon=10$ took into account the effect of the medium used in EPR experiments ${ }^{35}$ (ethyl acetate with 5\% acetonitrile vol/vol). Increased integration accuracy (Grid6, GridX8 and IntAcc 6.0 in ORCA convention) and tight SCF convergence criteria were applied.

Broken symmetry (BS) solutions for each optimized geometry are generated by inverting the local spin of individual Mn sites using the "FlipSpin" feature of ORCA. The number of computed energy differences between all possible single-determinant solutions is greater than the six possible pair-wise exchange coupling constants $J_{i j}$ between four Mn ions, so the system of linear equations is solved using singular value decomposition..$^{27}$ The exchange coupling constants are then used in the diagonalization of the HDvV Hamiltonian

$$
\hat{H}_{\mathrm{HDvV}}=-2 \sum_{i<j} J_{i j} \hat{S}_{i} \hat{S}_{j}
$$

that yields the complete spectrum of spin eigenstates. The diagonalization of the HDvV Hamiltonian is performed with the orca_eca module of ORCA.52

The advancement of density matrix renormalization group (DMRG) ${ }^{3.76}$ has enabled in recent years the calculation of near-exact many-electron wave functions of large systems such as oligonuclear transition metal complexes ${ }^{17-\pi s}$ using unprecedented active spaces. In the present work self-consistent field density matrix renormalization group calculations (DMRG-CASSCF) were conducted with the BLOCK code ${ }^{5.7982}$ as interfaced with ORCA. Starting orbitals were obtained by separate localization of occupied and unoccupied spaces from quasi-restricted DFT orbitals using the Pipek-Mezey method. ${ }^{83}$ All $3 \mathrm{~d}$ orbitals of Mn ions and all 2p orbitals of $\mathrm{O}$ bridges (32 orbitals in total) were included in the active space. The orbitals were ordered automatically with the Fiedler vector method. ${ }^{8 * s}$ The number of renormalized states $M$ and the 
energy convergence were adjusted to ensure negligible discarded weight in the density matrix and to achieve final accuracy in relative energies of the order of $10^{-4} \mathrm{kcal} \mathrm{mol}^{-1}$.

\section{RESULTS AND DISCUSSION}

3.1. S, State Model. Full optimizations of complex 1 in its neutral state can lead to three isomeric forms (S1a, S1b, and S1c), which differ in hydrogen bonding patterns that involve carboxylate ligands (Figure S1). All structures have the same valence state distribution Mn1(III)Mn2(IV)-Mn3(IV)-Mn4(III), which is clearly indicated by the Mulliken spin populations on the Mn centers, 3.94-4.00 for $\mathrm{Mn}$ (III) ions (local high-spin $\mathrm{d}^{4}$ configuration) and 2.96-3.04 for Mn(IV) ions (local high-spin $\mathrm{d}^{3}$ configuration). Among these isomers, the model with H-bonding between the carboxylic hydrogens and oxygens of carboxylato- and oxo- ligands (S1c) is found to be by far the most stable energetically, $11.4 \mathrm{kcal} \mathrm{mol}^{-1}$ lower in energy than $\mathbf{S 1 b}$ and $28.1 \mathrm{kcal}$ mol-1 $^{-1}$ lower in energy than S1a. Model S1c resembles the crystal structure closely and shows better agreement in terms of RMSD with the crystallographic values compared to previously reported optimizations ${ }^{\text {sss }}$ specifically $0.373 \AA$ for all non-H atoms and $0.037 \AA$ for the inorganic core. We attribute the improvement in optimized parameters by almost a factor of two in RMSD to the different methodological choices made in the present study, in particular the better performing TPSSh functional, ${ }^{1,323,3.6,5460.90}$ the larger all-electron basis sets, the inclusion of dispersion corrections, and the direct treatment of scalar relativistic effects through the ZORA Hamiltonian. In view of this, we expect that the present optimized models should also be a more reliable basis for further studying the electronic structure and spectroscopic properties of the synthetic complex in its various states. 
(a)
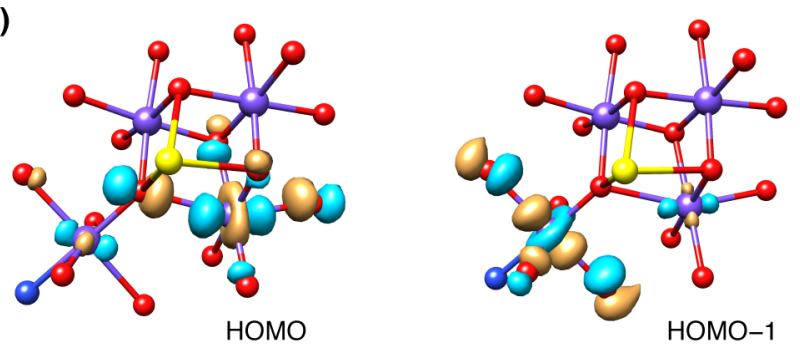

(b)

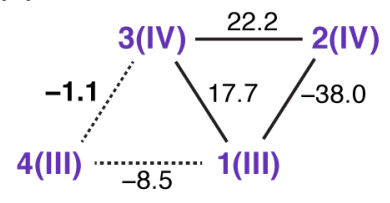

(c)

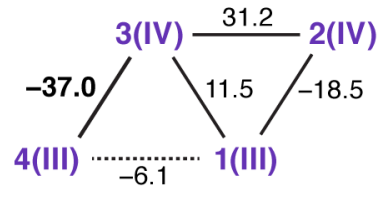

$$
S_{\mathrm{GS}}=1, S_{\mathrm{ES} 1}=0, S_{\mathrm{ES} 2}=2
$$$$
S_{\mathrm{GS}}=0, S_{\mathrm{ES} 1}=1, S_{\mathrm{ES} 2}=2
$$

$\Delta \mathrm{E} 1=1.2 \mathrm{~cm}^{-1}, \Delta \mathrm{E} 2=2.6 \mathrm{~cm}^{-1}$

Figure 3. a) Optimized core of model S1c, showing the two highest occupied molecular orbitals, HOMO and HOMO-1, localized on the Mn1(III) and Mn4(III) ions. b) Computed exchange coupling constants $\left(\mathrm{cm}^{-1}\right)$ for model S1c, with resulting three lowest spin states and their energy separation. c) Corresponding exchange coupling constants and associated parameters for a model of the $S_{1}$ state of the OEC (reoptimized with TPSS-D3BJ for the purposes of the present paper from a model reported by Krewald et al., ${ }^{12}$ using the same methods for magnetic properties as for the synthetic complex).

By inspection of the bond lengths in S1c, two pairs of Mn1-O and Mn4-O distances are seen to be clearly longer (by ca. $0.1 \AA$ on average) from other $\mathrm{Mn}-\mathrm{O}$ bonds, which indicates the orientation of the Jahn-Teller (JT) axes of the Mn1(III) and Mn4(III) ions. The orientation of JT axes corresponds to the nature of the frontier molecular orbitals: the HOMO and HOMO-1 of the complex are of $\mathrm{d}_{z^{2}}$ character predominantly localized on the Mn1(III) and Mn4(III) ions (Figure 3a) and are $\sigma$-antibonding with respect to the corresponding $\mathrm{O}$ atoms of the ligands. Compared with the $\mathrm{S}_{1}$ state of the OEC, the JT elongations here are less pronounced. For example, the Mn1 ion in the OEC is effectively five-coordinate as the bond with the internal oxo bridge $\mathrm{O} 5$ is practically broken. Importantly, in complex $\mathbf{1}$ the JT axis of Mn4 is oriented 
differently compared to the OEC, where a collinear (i.e. along the O5 bridge of the OEC, see Figure 1) as opposed to mutually perpendicular orientation of the JT axes of Mn1 and Mn4 was identified in the latest radiation-damage free XFEL crystallographic model of the $S_{1}$ state from Thermosynechococcus vulcanus (for discussions on and evaluations of this specific crystallographic model from various perspectives see Refs. ${ }^{\text {9.*4) }}$.

The pairwise exchange coupling constants as obtained through singular value decomposition are shown in Figure 3b. They agree in general with those reported by Shoji et al.s for the same state of the synthetic complex. Note that no single combination of spin alignments on Mn sites can satisfy the sign and magnitude of all exchange coupling constants, hence the model is an example of topological spin frustration ${ }^{* 5}$ typically associated with near-degeneracies of spin states. ${ }^{\circ 6}$ Indeed, in the present case the $S=1$ ground spin state is separated from the first excited $S$ $=0$ state and the second excited $S=2$ spin state by only $1.2 \mathrm{~cm}^{-1}$ and $2.6 \mathrm{~cm}^{-1}$, respectively.

The corresponding $\mathrm{S}_{1}$ state of the OEC, which has the same valence distribution as complex $\mathbf{1}$, is instead diamagnetic with a low-lying triplet state at ca. $2 \mathrm{~cm}^{-1.97}$ Computed exchange coupling constants for the $S_{1}$ state of the OEC are qualitatively similar to those computed for the synthetic complex, with the crucial exception of the coupling between Mn3 and the dangler Mn4 ion (Figure 3c). The additional oxo bridge between Mn3 and Mn4 in the OEC leads to strong antiferromagnetic coupling between the two ions similar to other states of the cluster, ${ }^{12}$ whereas $J_{34}$ in complex 1 is close to zero. Therefore, although the OEC and complex 1 resemble each other in the compression of low-lying spin states, the different bonding mode of the Mn4 in the synthetic complex results in a different magnetic topology as compared to the OEC, specifically in terms of the how $\mathrm{Mn} 4$ interacts with the cuboidal $\mathrm{Mn}_{3} \mathrm{CaO}_{4}$ unit. 
3.2. $\mathbf{S}_{2}$ State Isomeric Forms. Removal of one electron from the neutral form of complex $\mathbf{1}$ with Mn1(III)-Mn2(IV)-Mn3(IV)-Mn4(III) valence distribution can give rise to at least two oxidation state distributions, III-IV-IV-IV and IV-IV-IV-III, where the unique Mn(III) ion is located at either "end" of the cluster, similar to the interconvertible open and closed cubane forms in the $\mathrm{S}_{2}$ state of the OEC ${ }^{36}$ Efforts to optimize the one-electron oxidized form of complex $\mathbf{1}$ from different "guess" structures ultimately lead to these two isomeric forms $(\mathbf{S 2 a}$ and $\mathbf{S 2 d}$ in Figure 4). Additionally, we were able to converge and optimize the two other possible valence isomers, namely those that position the unique $\mathrm{Mn}(\mathrm{III})$ at the $\mathrm{Mn} 2$ or Mn3 site (models $\mathbf{S 2} \mathbf{b}$ and S2c). The position of the unique $\mathrm{Mn}(\mathrm{III})$ in these isomers is easily confirmed by a Mulliken spin population of 3.91-4.00, versus spin populations of 2.94-3.02 for the Mn(IV) ions. All of these forms are presented in Figure 4, along with their relative energies and the most relevant properties.

During optimization from various starting structures, a strong propensity of localizing Mn(III) at the dangling Mn4 was observed, in line with the fact that $\mathbf{S 2 d}$ is significantly more stable than all other isomers. The preferential oxidation of the Mn1 site of the $\mathrm{S}_{1}$ state, that is, of the $\mathrm{Mn}(\mathrm{III})$ ion that is located within the cuboidal unit, makes good chemical sense if one considers the molecular orbitals shown in Figure 3: the HOMO of the $S_{1}$ state is localized on Mn1, therefore the most accessible oxidation event is expected to be-and indeed it is - the oxidation of Mn1 to produce S2d. The HOMO-1 orbital in the $\mathrm{S}_{1}$ state thus becomes the HOMO of S2d. All structures that contain the $\mathrm{Mn}(\mathrm{III})$ ion within the cuboidal unit are significantly higher in energy, from 8.6 and $10.1 \mathrm{kcal} \mathrm{mol}^{-1}$ for positions Mn1 and Mn3 (models S2a and S2c) to $19.9 \mathrm{kcal} \mathrm{mol}^{-1}$ for Mn2 (model S2b). 
S2a
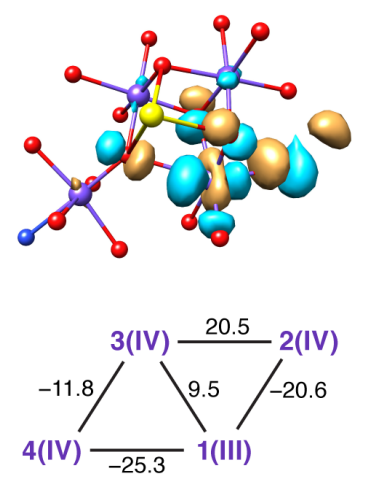

$E_{\text {rel }}=8.6 \mathrm{kcal} / \mathrm{mol}$,

$S_{\mathrm{GS}}=3 / 2, S_{\mathrm{ES}}=5 / 2, \Delta \mathrm{E}=9 \mathrm{~cm}^{-1}$
S2b
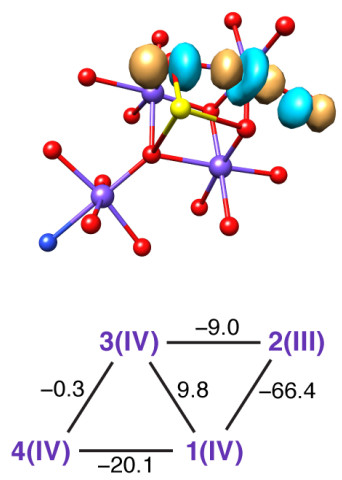

$E_{\mathrm{rel}}=19.9 \mathrm{kcal} / \mathrm{mol}$,

$S_{\mathrm{GS}}=1 / 2, S_{\mathrm{ES}}=3 / 2, \Delta \mathrm{E}=21 \mathrm{~cm}^{-1}$
S2c
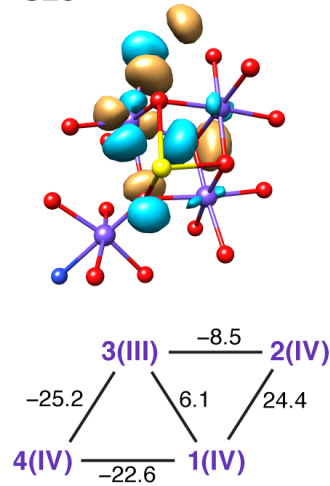

$E_{\text {rel }}=10.1 \mathrm{kcal} / \mathrm{mol}$,

$S_{\mathrm{GS}}=7 / 2, S_{\mathrm{ES}}=5 / 2, \Delta \mathrm{E}=4 \mathrm{~cm}^{-1}$
S2d
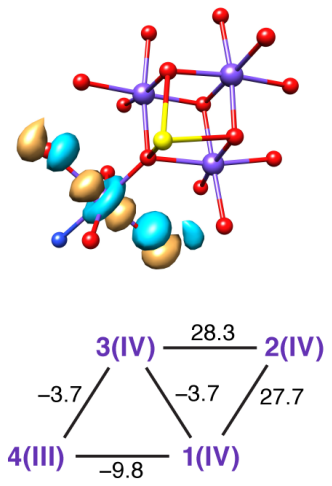

$E_{\text {rel }}=0.0 \mathrm{kcal} / \mathrm{mol}$,

$S_{\mathrm{GS}}=5 / 2, S_{\mathrm{ES}}=7 / 2, \Delta \mathrm{E}=34 \mathrm{~cm}^{-1}$

Figure 4. Highest occupied molecular orbitals of the one-electron oxidized forms of complex 1 (all HOMOs are centered on the unique Mn(III) ion of each model and the orientation of major antibonding interactions identifies the orientation of Jahn-Teller distortion), oxidation state distributions, exchange coupling constants $\left(\mathrm{cm}^{-1}\right)$, relative energies, spin of the ground $\left(S_{\mathrm{Gs}}\right)$ and first excited $\left(S_{\mathrm{ES}}\right)$ states and their energy separation for the four valence isomers.

Focusing on the two isomeric forms with IV-IV-IV-III (S2d) and III-IV-IV-IV (S2a) valence distributions that resemble the $S_{2}$ state forms of the OEC, we note that the energy difference between them is an order of magnitude larger than the ca. $1 \mathrm{kcal} \mathrm{mol}^{-1}$ difference between the corresponding pair of valence isomers in the OEC. ${ }^{3637}$ The relative stability of the two forms is also the reverse, because the III-IV-IV-IV form is the more stable form of the OEC in its $\mathrm{S}_{2}$ state, whereas for complex 1 the IV-IV-IV-III (S2d) form is by far the most stable.

In terms of structure, S2a resembles the open cubane $S_{2}$ state isomer of the OEC in the orientation of the Jahn-Teller axis of Mn1. However, there is a crucial difference for the S2d form, which would correspond to the closed cubane valence isomer of the OEC. Specifically, S2d maintains the Mn4 Jahn-Teller axis orientation of the parent S1c structure (Figure 3), i.e. the Jahn-Teller axis is perpendicular to the Mn4-O4 vector of complex 1, whereas in the corresponding closed cubane valence isomer of the OEC the Jahn-Teller axis of Mn4 lies along 
the $\mathrm{O} 5$ bridge. Moreover, in complex 1, the elongation of $\mathrm{Mn}-\mathrm{O}$ bond due to the Jahn-Teller effect is only ca. $0.5 \AA$, significantly smaller than the elongation of ca. $1.4 \AA$ in the OEC. Therefore, not only these two isomers in the $\mathrm{S}_{2}$ state of the synthetic complex are separated by a much larger energy difference than the $\mathrm{S}_{2}$ state isomers of the OEC, but also the different mutual orientation of Jahn-Teller axes in the $\mathbf{S 2 a} / \mathbf{S 2 d}$ isomers of the synthetic complex does not impart any flexibility in the coordination of the $\mathrm{O} 4$ bridge that is positioned between $\mathrm{Mn} 1$ and $\mathrm{Mn} 4$, and hence precludes the valence interconversion that is the hallmark of the OEC. ${ }^{36}$

The value and sign of exchange coupling constants in the isomeric pair S2a and S2d are similar to those in open and closed cubane structures of the OEC, but the coupling between $\mathrm{Mn} 1$ and Mn4 in complex $\mathbf{1}$ is significantly stronger than the coupling between the terminal ions in the OEC, presumably due to the more compact topology and the rigid Mn1-O4-Mn4 connectivity in complex 1. The large values of $J_{12}, J_{23}$ and $J_{34}$ in the $\mathbf{S 2 a}$ isomer support the $\alpha \beta \beta \alpha$ spin alignment, just like the open cubane $S_{2}$ form of the OEC, but this configuration is frustrated by the antiferromagnetic interaction between $\mathrm{Mn} 1$ and $\mathrm{Mn} 4$. This is in contrast to the OEC where the very weak coupling between Mn1 and Mn4 in the open cubane form does not give rise to competing interactions. This difference is ultimately reflected on the stabilization of higher-spin states for S2a ( $S=3 / 2$ ground spin state and $S=5 / 2$ first excited state), at variance with the $S=$ $1 / 2$ ground state of the open cubane form of the OEC. On the other hand, the S2d isomer has the same sextet ground spin state as the closed cubane $\mathrm{S}_{2}$ form of the OEC.

The HOMO of the S2b isomer is a Mn2-centered $\mathrm{d}_{z^{2}}$ orbital, whereas in the $\mathbf{S 2 c}$ isomer the HOMO that is localized on Mn3(III) has predominant $d_{x^{2}-y^{2}}$ character (Figure 4). The unusual occupancy of the $\mathrm{d}_{x^{2}-y^{2}}$ orbital in S2c is supported by the elongation of three Mn3-O bonds. The signs of exchange coupling constants between all ion pairs can be satisfied by $\alpha \beta \alpha \beta$ and $\alpha \alpha \alpha \beta$ 
type spin alignment in $\mathbf{S 2 b}$ and $\mathbf{S 2 c}$ isomers respectively, with the exception of $J_{23}$ in $\mathbf{S 2 c}$. The exchange coupling topology in $\mathbf{S 2 b}$ gives rise to the spin 1/2 ground state of this isomer, where as the S2c state is found to exist in a spin $7 / 2$ spin state commensurate with $\alpha \alpha \alpha \beta$ spin configuration. The doublet ground spin state in the $\mathbf{S 2} \mathbf{b}$ isomer is well separated from the first excited spin $3 / 2$ state by $21 \mathrm{~cm}^{-1}$, which is reminiscent of open cubane models for the $\mathrm{S}_{2}$ state of the OEC. However, as in the $\mathrm{S}$ models, the exchange coupling constant between Mn3 and Mn4 in the S2b form indicates the absence of superexchange interaction. In any case the model does not appear relevant for interpreting experiment due to its very high relative energy that would render it inaccessible.

Based on the above results, we can identify a structural and electronic analogy of the $\mathbf{S 2 d}$ ( $S=$ $5 / 2$ ) isomeric form of complex 1 with the closed cubane $S_{2}$ state form of the OEC. As we will show in the following, the experimental $g \approx 4.9$ EPR signal in the one electron oxidized form of complex $\mathbf{1}$ can be attributed to the $\mathbf{S 2 d}$ form. On the other hand, among the structures presented above there exists no low-spin isomer of comparable energy to $\mathbf{S 2 d}$, therefore we cannot yet suggest any form that would be analogous to the major component of the OEC that is the open cubane $S=1 / 2(g \approx 2$ multiline) form.

3.3. Refined Energetics. The large energy difference between the S2a and S2d forms as well as the absence of an energetically accessible spin $1 / 2$ state in the one-electron oxidized form of the synthetic complex contradict a recent computational study by Shoji et al.,8 who reported two almost isoenergetic forms that correspond to the S2a and S2d models described above. An energy separation of only $0.7 \mathrm{kcal}^{\text {mol-1 }}{ }^{-1}$ between these two isomeric forms was reported, which might allow the forms to coexist like the $\mathrm{S}_{2}$ state isomers of the OEC. Moreover, a spin state of $S$ $=1 / 2$ was attributed to a model that corresponds to isomer S2a of the present study. Taken 
together, these results could potentially satisfy the simultaneous observation of the $g \approx 2$ and $g \approx$ 4.9 EPR signals by invoking a situation analogous to the existence of interconvertible valence isomers in the OEC. Given this divergence of results, we investigated fully the origin of the discrepancy, which was eventually traced to the higher level of theory used in the present study.

Shoji et al. used the most stable broken-symmetry solution for the final geometry optimization of all species. Optimizing with a determinant that does not represent a spin eigenfunction is questionable as it might introduce bias towards a particular $M_{s}$ component. We confirm that optimization using a broken-symmetry determinant for $\mathbf{S 2} \mathbf{a}$ can influence the relative energies of the other broken-symmetry and the high-spin states sufficiently to result in a ground spin state of $S=1 / 2$. In our opinion this should be considered an artifact produced by biasing the calculation towards a particular spin alignment. Indeed, as pointed out in a recent comprehensive computational study of all S states of the OEC and of many oligonuclear manganese clusters, geometry optimization at the high spin state is free from such errors and has never failed to produce the correct ground spin state for any high-valent manganese complex of known structure..$^{12}$ Regardless of the predicted spin state, this technical point is of secondary importance to the fact that no matter how the optimization is carried out, the energy difference we compute between $\mathbf{S 2 a}$ and $\mathbf{S 2 d}$ remains greater than $8 \mathrm{kcal} \mathrm{mol}^{-1}$.

We concentrate therefore on understanding how a difference of less than $1 \mathrm{kcal} \mathrm{mol}^{-1}$ between the S2a and S2d valence isomers could be obtained by Shoji et al. The discrepancy does not appear to arise from a difference in optimized geometries, because application of our methods to the geometries published by Shoji et al. still leads to a similarly large energy difference as that reported here, $8.3 \mathrm{kcal} \mathrm{mol}^{-1}$. Therefore the difference comes from some aspect of the theoretical methods. We confirmed using the TPSSh functional that the ZORA-def2-TZVP basis set we are 
employing produces converged results by comparison with the largest member of the family, the quadruple-zeta polarized ZORA-def2-QZVP. There is no difference in relative energies of S2a and S2d obtained by the two basis sets so the results practically represent the basis set limit already with the polarized relativistically recontracted triple-zeta basis. Subsequently, we tested the performance of different DFT methods with the ZORA-def2-TZVP basis set. The results summarized in Table 1 very clearly indicate that despite variation among DFT methods, the S2a form remains energetically well separated from the S2d ground state isomer regardless of the choice of functional. The highest level of DFT methodology used in the present study is represented by the double-hybrid ${ }^{98-99}$ functionals B2PLYP, ${ }^{100}$ PWPB95, ${ }^{101}$ and B2GP-PLYP, ${ }^{102}$ which introduce a second order perturbative correction obtained with Møller-Plesset correlation treatment of the DFT orbitals. These methods still place the energy difference in the range 7.6$10.4 \mathrm{kcal} \mathrm{mol}^{-1}$, in agreement with the original TPSSh values.

By permuting various settings we excluded factors such as the treatment of relativity (ZORA

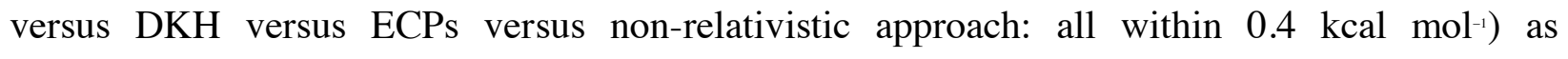
contributing factors, and eventually identified dispersion corrections (included in the present work through the D3BJ model but absent from the work of Shoji et al.) as the principal reason for the discrepancy. Dispersion corrections form a significant part of the total energy difference (see Table S1). Interestingly, in the case of our single-point calculations on the Shoji et al. geometries, even though the total energy difference we obtain with our methods is similar to the one we report here, the dispersion corrections have a larger contribution $\left(5.2 \mathrm{kcal} \mathrm{mol}^{-1}\right.$ of a total of $8.3 \mathrm{kcal} \mathrm{mol}^{-1}$ for TPSSh). If we use identical methods as those used by Shoji et al. (B3LYP with a combination of LANL2DZ and 6-31G* basis sets) the energy difference is only $1.1 \mathrm{kcal}$ $\mathrm{mol}^{-1}$, but adding D3BJ corrections increases the gap to $8.2 \mathrm{kcal} \mathrm{mol}^{-1}$. These results show that 
dispersion corrections are important and should, ideally, be included self-consistently as in the present work. Importantly, for methods that already account to some extent for dispersion effects these corrections are limited (Table S1), confirming that better methods already capture the physics correctly into the electronic energy and proving that the larger contributions obtained for lower-level functionals are not artifacts of the D3 model but physically meaningful corrections of the intrinsic deficiencies of the functionals. In conclusion, these results suggest that the abnormally low energy difference between species S2a and S2d reported by Shoji et al. was due to the lower level of theory and we conclude that only one form, the $S=5 / 2 \mathbf{S 2 d}$ isomer with $\mathrm{Mn}(\mathrm{III})$ positioned at the dangler Mn4 site, represents an energetically accessible one-electron oxidized form of complex $\mathbf{1}$.

Table 1. Energy difference ( $\mathrm{kcal} \mathrm{mol}^{-1}$ ) between the S2a and S2d isomers of complex $\mathbf{1}$ with different density functionals. Both structures for this specific comparison are optimized at their most stable broken symmetry state, $\mid \alpha \alpha \alpha \beta>\left(M_{s}=5 / 2\right)$ and $\mid \alpha \beta \alpha \beta>\left(M_{s}=1 / 2\right)$ respectively. Settings in terms of dispersion, relativity, basis sets, and integration accuracy follow those described in the Methodology section.

\begin{tabular}{lc}
\hline Functional & $\Delta E$ \\
\hline TPSSh & 8.5 \\
B3LYP & 6.9 \\
B3LYP* & 6.4 \\
B3PW91 & 9.1 \\
PBE0 & 9.0 \\
M06 & 10.1 \\
CAM-B3LYP & 8.2 \\
B2PLYP & 7.6 \\
PWPB95 & 10.4 \\
B2GP-PLYP & 9.1 \\
\hline
\end{tabular}

Using D3zero instead of D3BJ dispersion corrections. 
The energy difference between $\mathbf{S 2 a}$ and $\mathbf{S 2 d}\left(\Delta E=8.5 \mathrm{kcal} \mathrm{mol}^{-1}\right.$ for TPSSh) increases slightly when zero-point energy corrections are taken into account $\left(\Delta E=9.3 \mathrm{kcal} \mathrm{mol}^{-1}\right)$, and further when free energies are considered $\left(\Delta E=11.0 \mathrm{kcal} \mathrm{mol}^{-1}, T=298.15 \mathrm{~K}\right)$ through standard thermodynamic expressions on the basis of harmonic vibrational frequency calculations.

Despite the agreement of all DFT methods regarding the energy difference between the S2a and S2d isomers, it is useful to go beyond DFT in order to confirm the conclusion regarding the invalidity of the valence isomer hypothesis as an explanation of the two signals in the EPR spectrum of complex 1. We thus conducted multireference DMRG-CASSCF calculations on the two isomers both with a valence active space of 37 electrons in 32 orbitals (all Mn $3 \mathrm{~d}$ and all O $2 p$ orbitals), in analogy with the pioneering DMRG study of the OEC by Kurashige et al. ${ }^{77}$ The active space covers all main classes of configurations that contribute significantly to the many body wave function. ${ }^{103-106}$ The energy difference predicted by DMRG is $13.4 \mathrm{kcal} \mathrm{mol}^{-1}$ in favor of S2d (energies are converged to within $6 \times 10^{-4} \mathrm{kcal} \mathrm{mol}^{-1}$ with $M=500$ and discarded weight of the order of $10^{-15}$ ). Although further minor refinements of this value might be possible, the result is in clear and strong support of the DFT-derived conclusions presented above.

It is important to stress that the results obtained in the present work are not in conflict with the experimental data. ${ }^{35}$ Our results do not imply in any way that the multiline signal observed in EPR is an "impurity" of some sort, and do not call into question the validity of the data. Our conclusion is simply that the valence-isomer hypothesis regarding the origin of the two signals is most probably incorrect, and hence the structures that give rise to the two signals do not have the same mutual relationship as the two valence isomers that give rise to the two EPR signals in the $\mathrm{S}_{2}$ state of the OEC. The experimental data already suggest that this conclusion is reasonable, 
because the two signals that appear in the EPR spectrum of the synthetic complex are not interconvertible: given that the two relevant valence isomers S2a and S2d (Figure 4) are very similar structurally, regardless of the energy difference between them it should be expected that valence exchange between the terminal $\mathrm{Mn}$ ions must proceed considerably faster than in the OEC, where the intervening O5 bridge has to be relocated following a "breathing" movement of the cluster. ${ }^{36-37}$ However the study of Zhang et al. establishes that such interconversion is not even possible. ${ }^{35}$ In our view, this observation already speaks against the valence isomer hypothesis ${ }^{88}$ even without the evidence from our refined energetics that more conclusively disfavor this scenario. Of course, it must be recognized that the validity of the above analysis is contingent upon the ligands of the first coordination sphere in the one-electron oxidized form of the complex retaining the same coordination modes as in the neutral state.

3.4. Structural Variants for the $\mathbf{S}_{2}$ state. The results and analysis presented above suggest that the low-energy $S=5 / 2$ form of complex $\mathbf{1}$ in its $S_{2}$ state (model S2d) can explain the EPR signal observed at $g \approx 4.9$, but there exists no low-energy/low-spin form of the complex in this state that could explain the multiline signal at $g \approx 2$. This conclusion holds on the provision that the complex remains structurally intact upon oxidation. Therefore, to explain the emergence of the $g \approx 2$ multiline signal it appears necessary to invoke some form of structural change. It cannot be determined a priori whether this would be a minor structural modification/rearrangement, or a profound reorganization/decomposition of the oxidized complex. We point out however that the structure of the multiline EPR signal does suggest the presence of a magnetically coupled system containing more than two interacting Mn ions. In the following, without aspiring to be comprehensive, we explore a few hypothetical modifications that might provide clues about the origin of this signal. 
3.4.1. Protonation. It is possible that different valence isomers may be stabilized by different protonation patterns. Additionally, variable protonation of oxo bridges is the kind of minor perturbation that might leave the connectivity of the cluster unaffected but modify exchange coupling constants, alter the spacing or ordering of low-lying spin states, reorient JT axes, or even change the valence state distribution. ${ }^{12.50 .59}$ Here we investigated various protonated isomers of complex $\mathbf{1}$ in the $\mathrm{S}_{2}$ state, created by redistribution of carboxylic hydrogens within the complex. Figure S2 lists twenty of the structures considered, along with their ground spin states and relative energies with respect to $\mathbf{S 2 d}$. The results can be briefly summarized as follows: a) bridge protonation does not alter the doublet and sextet ground spin state in S2b and S2d isomeric forms, that is, proton shifts cannot turn $\mathbf{S 2 d}$ into a ground-state doublet, b) S2c and S2a are more sensitive and may change their spin state upon protonation of oxo bridges, and c) most importantly, all protonation isomers are without exception higher in energy than their parent structures, and hence all of them are even higher in energy compared to the global minimum S2d. Therefore, these protonation isomers will not be considered further.

3.4.2. Decomposition. It is well known that molecular water oxidizing catalysts often do not maintain their structural integrity and are deactivated through various decomposition pathways. ${ }^{107}$

${ }^{108}$ With this in mind, we attempted to model some structures, which might result from structural rearrangements of complex $\mathbf{1}$ upon oxidation. A few plausible deformed or decomposed structures of complex $\mathbf{1}$ in its $\mathrm{S}_{2}$ state are shown in Figure S3, along with their oxidation state distributions and ground spin states. Among the structures that could be obtained through loss of Ca or the dangler Mn, models that involve dissociation of calcium $(\mathbf{G}$ and $\mathbf{H}$ from $\mathbf{S 2 a}$ and $\mathbf{S 2 d}$ respectively) were inspired by EPR evidence which suggests that the basic electronic structure of the OEC is not perturbed by $\mathrm{Ca}^{2+}$ removal..$^{109}$ These structures exhibit different ground spin states 
compared to the intact S2a and S2d species, but are both high-spin $(S=5 / 2$ for $\mathbf{G}$ and $S=9 / 2$ for H). Models containing only three $\mathrm{Mn}$ ions were also evaluated on the assumption of decoordination of the dangling Mn: model I mimics the cubane model of Kanady et al.,27 and model $\mathbf{J}$ mimics the complex of Mukherjee et al., which contains an additional $\mathrm{Ca}^{2+}$ ion..$^{10}$ All of these models however exhibit strong ferromagnetic interactions resulting in the maximum spin state of $S=9 / 2$, similar to the original synthetic models. ${ }^{32}$

3.4.3. Rearrangement. In models $\mathbf{A}, \mathbf{B}, \mathbf{C}$ and $\mathbf{D}$ either the connectivity of carboxylate bridges around $\mathrm{Mn}$ ions or the distance between $\mathrm{Mn} 1$ and $\mu-\mathrm{O}_{4}$ are distorted so as to increase the Mn1Mn4 distance, which might result in reduced value of $J_{14}$ leading to a $S=1 / 2$ ground state. However, models $\mathbf{B}, \mathbf{C}$, and $\mathbf{D}$ persist in the same $S=5 / 2$ ground state as in intact $\mathbf{S 2 d}$, while model $\mathbf{A}$ has an even higher $S=11 / 2$ ground state. In another class of structures, represented by model $\mathbf{E}$ of Figure S3, the pyridine ligand attached to Mn4 is substituted by $\mathrm{CN}$. This change in the Mn4 coordination sphere does not result in change of spin states for either S2a or S2d.

A more drastic structural change was inspired by the butterfly structure of another mimic of the OEC reported by Dismukes and coworkers, "I' which led to the construction of model $\mathbf{F}$. In this model each pair of $\mathrm{Mn}$ ions are connected by at least one oxo and one carboxylate ligand to assure similarity in terms of the immediate coordination environment with the original structure of complex 1 , but otherwise the cube is unfolded. This is the only structure that leads to a $S=1 / 2$ ground state; on the other hand it is $44 \mathrm{kcal} \mathrm{mol}^{-1}$ higher in energy than $\mathbf{S 2 d}$ and hence should not represent a favorable rearrangement.

The above hypothetical deformed/decomposed derivatives of complex 1 offer motivation for interesting observations into magnetostructural correlations, but the crucial question here is if any of these transformations can offer a way towards explaining the existing observations. We 
cannot claim to have explored the chemical space of structural rearrangements extensively, but we can suggest that unless an energetically accessible rearrangement occurs that can enhance antiferromagnetic coupling, such as by "opening up" the cuboidal unit like in model $\mathbf{F}$, there is no obvious way to achieve an $S=1 / 2$ state energetically close to the most stable $S=5 / 2$ S2d form. In conclusion, the structural origin of the $g \approx 2$ EPR signal that appears upon oxidation of complex 1 remains uncertain, but we can assert that this signal does not arise from a simple valence isomer of the $\mathbf{S 2 d}$ form.

3.5. $\mathbf{S}_{3}$ State. For completeness we also constructed a model of the two-electron oxidized form $\left(\mathrm{S}_{3}\right.$ state) of complex $\mathbf{1}$, using the structure of the $\mathrm{S}_{2}$ state as a starting point. The optimized structure shows all $\mathrm{Mn}-\mathrm{O}$ bonds to have very similar lengths of ca. $1.9 \AA$, consistent with absence of any Jahn-Teller distortion, and the spin populations of the Mn ions (2.90-3.03) confirm that all Mn ions are present as Mn(IV). The ground state of this two-electron oxidized form of complex 1 is $S=3$ with a large separation $\left(40.2 \mathrm{~cm}^{-1}\right)$ from the first excited $S=2$ state. These observations are similar to the findings for the $S_{3}$ state of the OEC..$^{10}$ These results suggest that magnetic data (EPR, $\left.{ }^{5} \mathrm{Mn}-\mathrm{ENDOR}\right)$ of the two-electron oxidized structure could be particularly valuable, especially towards examining any putative substrate/solvent interactions.

3.6. Redox Potentials. The cyclic voltammetry data on complex $\mathbf{1}$ and its structural similarity with the OEC make the model complex a valuable reference for understanding the interplay between structure and electrochemical behavior. Recent experimental studies on a series of heterobimetallic cubanes provide hints about the possible roles of the calcium ion in the OEC. ${ }^{30-}$ 31, 34 Quantum chemical studies on the same series exactly reproduced the experimentally observed correlation between the redox potential and the Lewis acidities of redox-inactive cations, revealing however at the same time that the OEC and the synthetic models have 
fundamentally distinct responses to the nature of the redox-inactive cation. ${ }^{33}$ Following the computational strategy used in this and other theoretical studies on the redox behavior of oligonuclear Mn clusters, ${ }^{33,112}$ the relative redox potentials for complex $\mathbf{1}$ are computed in terms of the total energy difference $(\Delta E)$ between the oxidized and reduced species. The energy used for comparison with experiment thus includes the electronic energy and the solvation free energy contributed by a continuum solvation model. ${ }^{113}$ According to Ho, the direct use of implicit solvation energies over thermodynamic cycles is preferable because it avoids problems arising from the imbalanced treatment of geometric and electronic structures of charged species in the gas phase and in solution. ${ }^{114}$ For the present purposes, reduced states of the synthetic complex $\mathbf{1}$, corresponding to $\mathrm{S}_{0}$ and $\mathrm{S}_{-1}$, are optimized with a total charge of -1 and -2 using the same methods as for the other states. The $\mathrm{S}_{0}$ state adopts the oxidation states $\mathrm{Mn}(\mathrm{III})_{3} \mathrm{Mn}(\mathrm{IV})$, as in the $\mathrm{S}_{0}$ state of the OEC, with two isoenergetic valence distributions, III-IV-III-III and IV-III-III-III. The most stable form we have located for the $\mathrm{S}_{-1}$ state has an oxidation state distribution of IIIIV-III-II, placing $\mathrm{Mn}(\mathrm{II})$ at the dangling position. This is in contrast to the assumption of an all$\mathrm{Mn}(\mathrm{III})$ species shown by Zhang et al. ${ }^{35}$ We note that coexistence of three distinct Mn oxidation states within the same cluster has a synthetic precedence. ${ }^{115}$ Using the most stable species that could be identified for each oxidation state of the complex, energy differences were computed with two implicit solvation models, COSMO and SMD and the results are correlated with the experimentally estimated midpoint potentials for the transitions in Figure 5. Both solvation models yield $\mathrm{R}^{2}=0.98$ for the correlation between computed $\Delta E$ values and midpoint potentials, showing that the approach is robust and that the reliability of the predictions depend less on the free energy corrections by the solvation model and more on the description of the electronic structure of the different oxidation states of the complex. 


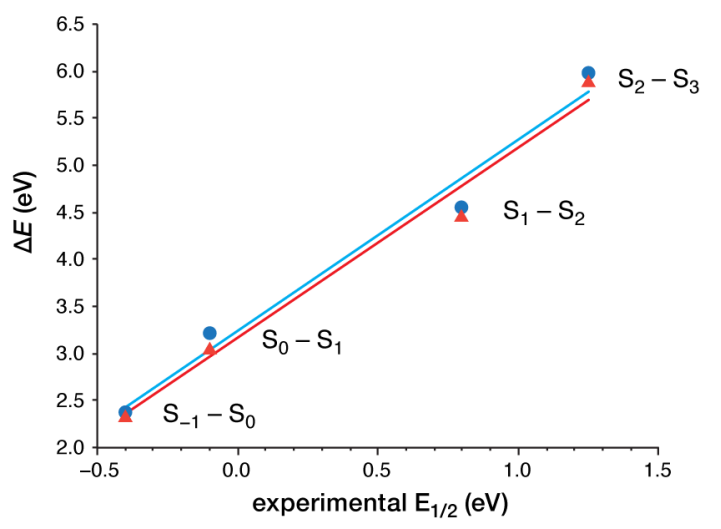

Figure 5. Correlation of computed energy differences for the individual $S_{n}-S_{n+1}$ transitions of complex 1 using two different continuum solvation models, COSMO (blue circles) and SMD (red triangles) with the experimentally derived midpoint potentials.

3.7. EPR Simulations. The above results on complex 1 shed light on how to interpret low frequency (X/Q-band) EPR spectra of high spin states of the OEC, particularly the parallel polarized EPR signals seen for the $\mathrm{S}_{1}$ state, allowing the information content of these earlier studies to be fully realized. Figure 6 shows a summary of the reported high spin EPR signals seen for complex $\mathbf{1}$ in both its as-isolated (" $\mathrm{S}_{1}$ state") and one-electron oxidized form, compared to literature EPR data for the higher plant OEC in its $S_{1}$ and $S_{2}$ states. ${ }^{44,} 116-117$ While the respective signals for the two systems are similar, it is the differences betweent the two sets of spectra that are instructive. As both have well characterized structural data, complemented by theoretical modelling, changes in the extact shape and position of the EPR signals seen for these two systems can now be directly correlated to structural differnces. This has important consequences for the interpretation of high spin signals for the higher S-state intermediates of the OEC, for which good crystallographic data is not yet available, providing a means to monitor structural evolution through the catalytic cycle. ${ }^{10,12-13,47-48}$ 
EPR signals that arise from high spin, non-integer transition metal complexes are typically very broad, resolving turning points far from the free-electron spectral position $(g=2.0028, \mathrm{X}$ band/9.6 GHz $=0.34 \mathrm{~T}$ ). For many systems the intrinsic magnetic interactions of the complex, derived from spin-spin and spin-orbit coupling of the set of unpaired electrons, are much larger than the interaction with the external magnetic field, i.e. the Zeeman interaction (X-band/9.6 $\left.\mathrm{GHz}=0.3 \mathrm{~cm}^{-1}\right)$. In this instance, as is the case for complex $\mathbf{1}$ in its one-electron oxidized state, the width of the spectrum is defined by the fine structure parameter $(D)$, which describes the magnetic splitting(s) of the complex at zero-applied magnetic field (ZFS - zero-field splitting). The exact shape of the signal reflects the symmetry of the fine structure tensor, which is described by the rhombicity parameter $E$. It is typically reported as the dimensionless parameter $E / D$, with unique range of $0 \leq E / D \leq 0.33$. A further complication arises for high spin, noninteger transition metal complexes, of which complex $\mathbf{1}$ in its as-isolated form is representative. Such complexes often do not display Kramers degeneracy and thus cannot be measured using conventional EPR at the low microwave frequencies, for example X-band. They can, however, be observed using parallel mode EPR, which allows the pumping of spin forbidden transitions of the spin manifold - transitions between pseudo-Kramers states. Such transitions become partially allowed in the instance where the $D$ value is small and/or the rhombicity parameter is small. 

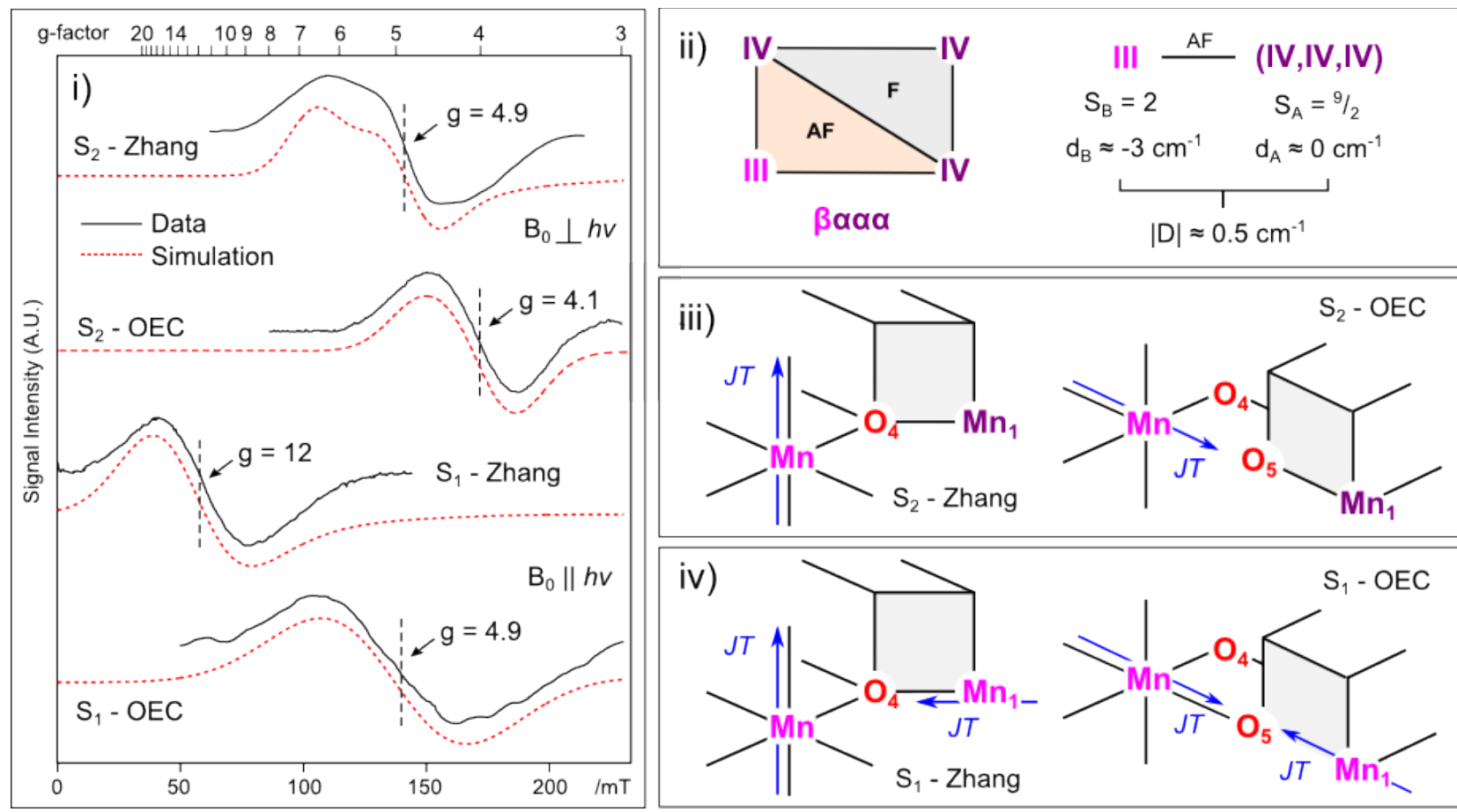

Figure 6. i) Comparison of X-band EPR spectra for complex 1 (Zhang) in its as-isolated and one-electron oxidized forms ${ }^{35}$ to the corresponding states for the OEC taken from refs ${ }^{16}$ Simulation parameters: $S_{2}$-Zhang $\left[S=5 / 2, D=0.5 \mathrm{~cm}^{-1}, E / D=0.035\right], \quad S_{2}-$ OEC $[S=5 / 2$, $\left.D=0.5 \mathrm{~cm}^{-1}, E / D=0.25\right], \mathrm{S}_{\mathrm{t}}$-Zhang $\left[S=1, D=1.0 \mathrm{~cm}^{-1}, E / D=0.15\right]$ and S,-OEC $[S=1$, $\left.D=0.14 \mathrm{~cm}^{-1}, E / D=0.20\right]$. ii) magnetic coupling scheme - decomposition of contributions to the total ZFS $(D)$ of the complex; iii and iv) orientations of the Jahn-Teller axis/es of the Mn(III) ion(s) in the one-electron oxidized and the as-isolated state. The Jahn-Teller axis is expected to coincide with the principal axis of the local Mn(III) fine structure tensor.

Spin Hamiltonian simulations of the EPR signals of complex $\mathbf{1}$ and the OEC were performed using the EasySpin package ${ }^{118}$ and are also shown in Figure 6. The one-electron oxidized form could be simulated as arising from an $S=5 / 2$ spin system. As expected, a $|D|$ value in excess of $0.4 \mathrm{~cm}^{-1}$ was required to rationalize the spectrum, of approximately axial symmetry (rhombicity parameter $E / D$ of 0.04$)$. To reproduce the precise structure of the signal, the rhombicity was modeled as a Gaussian distribution with FWHM of $200 \mathrm{MHz}$. As no hyperfine structure is observed, it can be inferred that the hyperfine constant $(A)$ for each Mn ion is less than the fitted 
linewidth $(28 \mathrm{mT}, 70 \mathrm{MHz})$. A simulation of the as-isolated form could also be achieved. Its single turning point at $g=12$ could be modeled as arising from an $S=1$ spin system. A wide range of $D$ and $E / D$ were able to reproduce the spectrum with the minimum $D$ value $\approx 0.5 \mathrm{~cm}^{-1}$.

The spin states inferred for complex $\mathbf{1}$ in its oxidized (" $\mathrm{S}_{2}$ state") and as-isolated (" $\mathrm{S}$, state") forms matched those of the DFT models developed in this study, S2d and S1c. Furthermore, the two DFT models also readily rationalize the magnitude of $D$ and its symmetry (the $E / D$ value). As described above, the total $D$ value is made up of contributions from all paramagnetic ions of the complex. ${ }^{111022}$ The oxidized form of the complex contains three $\mathrm{Mn}(\mathrm{IV})$ ions and one $\mathrm{Mn}(\mathrm{III})$ ion, as in the $\mathrm{S}_{2}$ state of the OEC. ${ }^{121,123 \cdot 126}$ In $\mathbf{S 2 d}$ the three $\mathrm{Mn}(\mathrm{IV})$ ions are all locally high-spin $(S=$ $3 / 2$ ), with an octahedral ligand field. Thus their local electronic structure is approximately spherically symmetric (half-filled $t_{28}$ orbital set, ${ }^{4} \mathrm{~A}_{28}$ state) which gives rise to only small local fine structure interaction $\left(d<0.3 \mathrm{~cm}^{-1}\right)^{121-123, \text {,20.128 }}$ which has a negligible contribution to the total $D$ value. ${ }^{21,123.124}$ Thus the $D$ value instead reflects the local fine structure parameter of the Jahn-Teller active $\mathrm{Mn}(\mathrm{III})$ ion, which has much larger local $d$ (ca. $3 \mathrm{~cm}^{-1}$ ) calibrated against model complexes. ${ }^{121-123,127-30}$ As the $\mathrm{Mn}(\mathrm{III})$ carries only part of the "total spin", this value scales to approximately $0.5 \mathrm{~cm}^{-1}$ for the tetramanganese complex. It is noted that the rhombicity $(E / D)$ of the complex also reflects the local e/d value for the $\mathrm{Mn}$ (III) ion. The small value seen for complex $\mathbf{1}$ is consistent with the $\mathrm{Mn}(\mathrm{III})$ ion (located at the dangler Mn4 position in the S2d isomer) having a symmetric six-coordinate quasi-octahedral ligand field, as seen in the crystal structure of the precursor state. ${ }^{35}$

The reduced form of the complex instead contains two Mn(IV) and two Mn(III) ions. As the complex now contains two transition metal ions that have a significant contribution to the total $D$ value, its value should in principle increase, i.e. approximately two-fold compared to that of the 
oxidized complex, as inferred from spin Hamiltonian simulations of the as-isolated complex 1. Importantly though, the additive contribution of the local $d$ values to the total $D$ only happens under certain circumstances, namely if the local $d$ values for each site have the same sign (i.e. the same local electronic state) and if the spin projection weightings also have the same sign. The first property can be immediately confirmed from the crystal structure of complex $\mathbf{1}$ and from the corresponding model S1c: the local electronic structure of both $\mathrm{Mn}(\mathrm{III})$ ions is indeed the same (the highest occupied orbital is of metal $\mathrm{d}_{z^{2}}$ character, i.e. both Mn ions would be of ${ }^{s} B_{\mathrm{Ig}}$ origin). The second property, that is, the spin projection weightings, comes about from the exchange coupling topology and can be calculated as described previously.$^{10}$ For the $\mathbf{S 2 d}$ model the spin projections for each site are the same and thus a large $D$ is expected. The large rhombicity parameter can also be explained by model S1c. In part this comes about because the two Mn(III) ions do not share the same reference frame, that is, their local $d$ tensors with principal axes aligned with the Jahn-Teller elongation axes are rotated by $90^{\circ}$ to each other. This has the consequence that different Cartesian components of each local tensor sum together to give the total $D$ value, leading to a large $E / D$ value.

As remarked in the original study of Zhang et al., ${ }^{35}$ the two EPR signals described above are remarkably similar to those observed for nature's oxygen-evolving complex. Multifrequency EPR measurements of the high spin $\mathrm{S}_{2}$ state $(g \geq 4.1),{ }^{117}$ along with SQUID data ${ }^{131}$ reveal that the spin state and $D$ value for the OEC is essentially the same, ca. $0.5 \mathrm{~cm}^{-1}$. However, the spin Hamiltonian simulations described above do illustrate a crucial difference between the two systems: the high spin $\mathrm{S}_{2}$ state of the OEC has a much larger $E / D$ value. This cannot be due to the coupling of the $\mathrm{Mn}(\mathrm{III})$ ion to the $\mathrm{Mn}_{3} \mathrm{O}_{4} \mathrm{Ca}$ cubane unit because a large $E / D$ value would then also be expected for complex 1. It instead indicates that the local ligand field of the Mn(III) ion 
of the OEC is different from that of complex 1. This is indeed expected: DFT models of the high spin $\mathrm{S}_{2}$ OEC suggest that the $\mathrm{Mn}(\mathrm{III})$ ion is not six-coordinate but instead represents a fivecoordinate square pyramidal site.$^{36}$ This is of key importance to the function of the cofactor as the availability of open coordination sites provides a putative substrate water binding site.

Complex 1 also provides new insight into the electronic structure of the $S_{1}$ state of the OEC. The EPR signal of the synthetic complex resembles the $S_{1}$ state parallel mode signal seen in spinach PSII, which resolves a single turning point at $g=4.9 .{ }^{116}$ The shift of the signal from $g=12$ in the synthetic complex to 4.9 in the biological system follows directly from the change in the coupling scheme and orientation of the local $d$ tensors of the two $\mathrm{Mn}(\mathrm{III})$ ions. Earlier simulations of this excited state signal fit the $g=4.9$ turning point to an $S=1$ spin state with very small $D$ and $E$ values. As in the model complex, the local electronic structure of the two $\mathrm{Mn}(\mathrm{III})$ ions is expected to be the same, but unlike the synthetic model the spin projection weightings for each site should be of opposite sign. As the local $d$ tensor of each Mn(III) for the OEC are collinear, with the Jahn-Teller axis of the two Mn(III) ions pointing in the same direction, ${ }^{7}$ this leads to the local $d$ values of the two Mn(III) ions cancelling each other out, leading to an unexpectedly small $D$ and $E$ value for the $\mathrm{S}_{1}$ state of the OEC. We note that the $\mathrm{S}_{1}$ signal seen in cyanobacterial PSII (P6803) has a more complicated structure, ${ }^{132}$ in part we suspect because it is less well described as an isolated excited spin state, as is the case for the $S_{1}$ signals in spinach. ${ }^{124}$

3.8. Superoxidized State and Relevance for Water Oxidation. In view of the similarities with the OEC and its ability to function as a redox accumulator, an important question is whether complex 1 can also function as a catalyst in water oxidation. Experimental data on this point are inconclusive, but according to the results presented here, we suggest that the answer is probably no. Oxidation of the $\mathrm{S}_{3}$ computational model reported above results in ligand-based oxidation 
regardless of the total spin multiplicity assumed for the complex. This indicates that it is not possible to oxidize any of the Mn(IV) ions further and is consistent with the absence of metalcentered frontier orbitals for the all-Mn(IV) species (see Figure S4). Oxidation instead results in formation of a radical on the carboxylate ligand that is bridging $\mathrm{Ca}$ and $\mathrm{Mn} 1$, as shown by the development of spin density on the ligand (Figure 7). This is accompanied by significant elongation of the $\mathrm{C}-\mathrm{C}$ bond (from $1.530 \AA$ to $1.918 \AA$ ), implying incipient bond breaking. We interpret this result as suggestive of ligand decomposition upon further oxidation of the $\mathrm{S}_{3}$ state. This is consistent with the cyclic voltammetry studies which showed the oxidation of the $\mathrm{S}_{3}$ state to be irreversible..$^{35}$
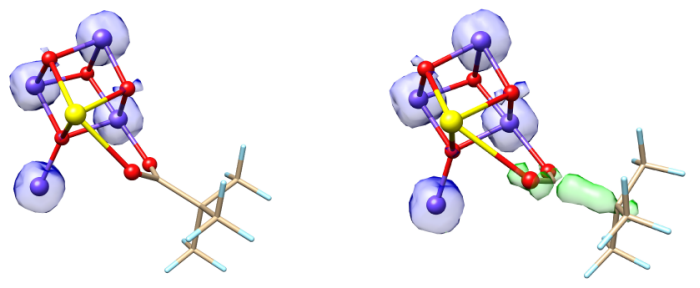

Figure 7. Comparison of spin density distribution between the $\mathrm{Mn}(\mathrm{IV})_{4} \mathrm{~S}_{3}$-state model of complex 1 (left) and its oxidized form (right). Blue and green color indicates majority and minory spin. All attempts to simulate a hypothetical $S_{4}$ state for the synthetic model result in ligand-based oxidation and $\mathrm{C}-\mathrm{C}$ bond breaking.

The implication is that there can be no oxidative progression that resembles the OEC, and no formation of a "hot" species that might be involved in $\mathrm{O}-\mathrm{O}$ bond formation. Possible mechanisms for this step include nucleophilic attack of $\mathrm{H}_{2} \mathrm{O} / \mathrm{OH}$ to a terminal $\mathrm{Mn}(\mathrm{V})$ oxo/Mn(IV)-oxyl, ${ }^{13-134}$ and radical coupling involving at least one terminal Mn(IV)-oxyl..$^{135-138}$ Neither type of activated oxo can be formed in complex $\mathbf{1}$ as there are no terminal water-derived ligands, i.e. complex 1 does not ligate any substrates in its as-isolated coordination environment. This, however, does not exclude the possibility that the complex represents a pre-catalyst. 
Indeed, Raucci et al. have described a hypothetical process in which they used a water substituted model of complex I, based upon the suggestion that the compound may slowly exchange its ligands upon slow addition of water. ${ }^{\text {s9 }}$ This study finds that the inserted water ligand between $\mathrm{Ca}$ and dangling $\mathrm{Mn} 4$ may participate in $\mathrm{O}-\mathrm{O}$ bond formation through a series of oxidation and deprotonation steps and thus highlights the importance of an additional oxo ligand between Mn3 and Mn4, which is missing in the complex 1. Although these computational results suggest that the inorganic core itself may in principle support water oxidation chemistry, the necessity for extensive ligand substitution shows that the original ligand environment is not compatible with such function.

\section{CONCLUSIONS}

The present work investigated the geometric and electronic structure of a tetramanganesecalcium complex reported by Zhang et $\mathrm{al}_{.^{35}}$ as the closest, at present, structural mimic of the oxygen-evolving complex of photosystem II. The complex was shown to access different oxidation states similar to the states of the Kok cycle in photosystem II..$^{35}$ Most intriguingly, the one-electron oxidized state of the complex was suggested to mimic the EPR properties of the $\mathrm{S}_{2}$ state of the biological catalyst as it was reported to give rise to two distinct signals at $g \approx 4.9$ and $g \approx 2,{ }^{, 3}$ reminiscent of the $g \geq 4.1$ and $g \approx 2$ signals associated with the $\mathrm{S}_{2}$ state of the OEC.

Our calculations and analysis suggest that the synthetic complex not only exhibits geometric but also electronic similarities with the OEC in the $S_{1}, S_{2}$ and $S_{3}$ states. The valence state distribution and the ground spin state in the $S_{1}$ and $S_{3}$ states are directly analogous to those in the OEC. However, the congruency between the synthetic complex and the OEC in the $S_{1}$ and $S_{3}$ states holds only partly for the $\mathrm{S}_{2}$ state. Among all isomeric forms of the one-electron oxidized 
state of the synthetic complex, an $S=5 / 2$ form (model S2d, with the Mn(III) ion located at the "dangler" position) emerges as the only energetically accessible isomer. This forms readily explains the appearance of the $g \approx 4.9$ EPR signal, which can be considered analogous to the $g \geq$ 4 signals associated with the $S_{2}$ state of the OEC. However, this is where the analogy with the biological cluster stops, because no valence isomer of the synthetic complex could be identified as the origin of the reported $g \approx 2$ multiline signal, considering both relative energies and spin states. This is supported by a range of methods including double-hybrid density functionals and accurate multireference density matrix renormalization group (DMRG) calculations, which confirm that an alternative valence isomer previously suggested ${ }^{88}$ as a potential candidate for the $g \approx 2$ form (III-IV-IV-IV) is not low enough in energy to coexist with the most stable $S=5 / 2$ (IV-IV-IV-III) isomer.

This leads us to conclude that the $g \approx 2$ EPR signal detected upon oxidation of the synthetic complex may be associated with an isomerization or rearrangement product. This suggestion is in fact consistent with the experimental observation that the two signals do not interconvert, ${ }^{35}$ in conflict with the valence-isomer hypothesis ${ }^{88}$ that implies negligible valence-shift barrier as a consequence of the structural similarity of the hypothetical valence isomers. Evaluation of different protonation isomers and potential rearrangement products did not provide any obvious candidate for a spin doublet form that is energetically close to the most stable $\mathbf{S 2 d}$ isomer, therefore the origin of this signal remains an open question. The appearance of two signals in the EPR spectrum of oxidized samples of complex 1 thus cannot be of similar origin to the valence isomerism encountered in the $\mathrm{S}_{2}$ state of the OEC. A defining feature of the latter is the conformationally fluxional oxo bridge that toggles between the terminal Mn ions as they swap oxidation states, ${ }^{36-37}$ the same oxo bridge suggested to be one of the substrates in $\mathrm{O}-\mathrm{O}$ bond 
formation. ${ }^{538-138,400142}$ This feature is not present in the rigid structure of complex $\mathbf{1}$ and has not been replicated by any synthetic analogue reported to date. The present results do not diminish the significance of the synthetic achievement represented by the structure of complex $\mathbf{1}$, but they do redefine the limits of how far the analogy to the OEC can be taken.

Nevertheless, complex $\mathbf{1}$ is of significant importance to the biological water splitting field, as demonstrated above when compared against literature EPR results for the OEC. In addition, the importance of high spin states involving the dangler $\mathrm{Mn}$ and a closed cubane structure have recently been highlighted by recent theoretical studies of the mechanism of water oxidation..$^{419}$ These studies have suggested that water access to the cofactor in the $S_{3}$ state is mediated by this type of intermediate structure, ,s. $^{\text {st }}$ through a channel that coincides with the access channel of substrate analogs such as methanol. ${ }^{50}$ It has also been suggested that the high spin topology of the cofactor may be important in imparting water oxidizing activity. ${ }^{13}$ The Zhang complex, together with the earlier models of Agapie and Christou, provide a framework as to how the magnetic properties of the whole complex are tuned by its constituent parts, i.e. the dangler $\mathrm{Mn}$ and the $\mathrm{Mn}_{3} \mathrm{O}_{4} \mathrm{Ca}$ cubane. The analysis we present here shows that the EPR spectra of the complex encode structural information on the coordination number and local electronic state of the dangler Mn, demonstrating that EPR may be the method of choice to test substrate uptake proposals which involve substrate binding via coordination to the dangler Mn site.

Supporting Information. Figures S1-S4 and Cartesian coordinates of optimized structures. This material is available free of charge via the Internet at http://pubs.acs.org.

\section{Corresponding Author}

*Email: dimitrios.pantazis@cec.mpg.de 


\section{ACKNOWLEDGMENTS}

Financial support by the Max Planck Society and by the project MANGAN (03EK3545) funded by the Bundesministeriums für Bildung und Forschung (BMBF) is gratefully acknowledged. NC acknowledges the support of the Australian Research Council (FT140100834). DP acknowledges network support by the COST action CM1305 "Explicit Control Over Spin-States in Technology and Biochemistry (ECOSTBio)". The authors thank Kantharuban Sivalingam and Yang Guo for helpful discussions regarding the DMRG calculations. 


\section{REFERENCES}

1. Blankenship, R. E. Molecular Mechanisms of Photosynthesis. Blackwell: Oxford, 2001; p 321.

2. Krewald, V.; Retegan, M.; Pantazis, D. A. Principles of Natural Photosynthesis. Top. Curr. Chem. 2016, 371, 23-48.

3. McEvoy, J. P.; Brudvig, G. W. Water-splitting chemistry of photosystem II. Chem. Rev. 2006, 106, 4455-4483.

4. Dau, H.; Limberg, C.; Reier, T.; Risch, M.; Roggan, S.; Strasser, P. The Mechanism of Water Oxidation: From Electrolysis via Homogeneous to Biological Catalysis. ChemCatChem 2010, 2, 724-761.

5. Cox, N.; Pantazis, D. A.; Neese, F.; Lubitz, W. Biological water oxidation. Acc. Chem. Res. 2013, 46, 1588-1596.

6. Umena, Y.; Kawakami, K.; Shen, J.-R.; Kamiya, N. Crystal structure of the oxygenevolving photosystem II at a resolution of $1.9 \AA$. Nature 2011, 473, 55-60.

7. Suga, M.; Akita, F.; Hirata, K.; Ueno, G.; Murakami, H.; Nakajima, Y.; Shimizu, T.; Yamashita, K.; Yamamoto, M.; Ago, H.; Shen, J.-R. Native structure of photosystem II at 1.95 A resolution viewed by femtosecond X-ray pulses. Nature 2015, 517, 99-103.

8. Yano, J.; Yachandra, V. $\mathrm{Mn}_{4} \mathrm{Ca}$ Cluster in Photosynthesis: Where and How Water is Oxidized to Dioxygen. Chem. Rev. 2014, 114, 4175-4205.

9. Kok, B.; Forbush, B.; McGloin, M. Cooperation of charges in photosynthetic $\mathrm{O}_{2}$ evolution - I. A linear four step mechanism. Photochem. Photobiol. 1970, 11, 457-475.

10. Cox, N.; Retegan, M.; Neese, F.; Pantazis, D. A.; Boussac, A.; Lubitz, W. Electronic structure of the oxygen- evolving complex in photosystem II prior to $\mathrm{O}-\mathrm{O}$ bond formation. Science 2014, 345, 804-808

11. Krewald, V.; Neese, F.; Pantazis, D. A. Resolving the Manganese Oxidation States in the Oxygen-evolving Catalyst of Natural Photosynthesis. Isr. J. Chem. 2015, 55, 1219-1232.

12. Krewald, V.; Retegan, M.; Cox, N.; Messinger, J.; Lubitz, W.; DeBeer, S.; Neese, F.; Pantazis, D. A. Metal oxidation states in biological water splitting. Chem. Sci. 2015, 6, 16761695.

13. Pérez-Navarro, M.; Neese, F.; Lubitz, W.; Pantazis, D. A.; Cox, N. Recent developments in biological water oxidation. Curr. Opin. Chem. Biol. 2016, 31, 113-119. 
14. Pantazis, D. A.; Cox, N.; Lubitz, W.; Neese, F., Oxygen-evolving Photosystem II. In Encyclopedia of Inorganic and Bioinorganic Chemistry, Scott, R. A., Ed. John Wiley \& Sons, Ltd: 2014; pp 1-13.

15. Lubitz, W.; Reijerse, E. J.; Messinger, J. Solar water-splitting into $\mathrm{H}_{2}$ and $\mathrm{O}_{2}$ : design principles of photosystem II and hydrogenases. Energy Environ. Sci. 2008, 1, 15-31.

16. Dau, H.; Zaharieva, I. Principles, Efficiency, and Blueprint Character of Solar-Energy Conversion in Photosynthetic Water Oxidation. Acc. Chem. Res. 2009, 42, 1861-1870.

17. Najafpour, M. M.; Govindjee. Oxygen evolving complex in Photosystem II: Better than excellent. Dalton Trans. 2011, 40, 9076-9084.

18. Wiechen, M.; Najafpour, M. M.; Allakhverdiev, S. I.; Spiccia, L. Water oxidation catalysis by manganese oxides: learning from evolution. Energy Environ. Sci. 2014, 7, 22032212.

19. Kurz, P. Biomimetic Water-Oxidation Catalysts: Manganese Oxides. Top. Curr. Chem. 2016, 371, 49-72.

20. Rivalta, I.; Brudvig, G. W.; Batista, V. S. Oxomanganese complexes for natural and artificial photosynthesis. Curr. Opin. Chem. Biol. 2012, 16, 11-18.

21. Blakemore, J. D.; Crabtree, R. H.; Brudvig, G. W. Molecular Catalysts for Water Oxidation. Chem. Rev. 2015, 115, 12974-3005.

22. Young, K. J.; Brennan, B. J.; Tagore, R.; Brudvig, G. W. Photosynthetic Water Oxidation: Insights from Manganese Model Chemistry. Acc. Chem. Res. 2015, 48, 567-574.

23. Brynda, M.; Britt, R. D., The Manganese-Calcium Cluster of the Oxygen-Evolving System: Synthetic Models, EPR Studies, and Electronic Structure Calculations. In Metals in Biology: Applications of High-Resolution EPR to Metalloenzymes, Hanson, G.; Berliner, L., Eds. Springer New York: New York, NY, 2010; pp 203-271.

24. Mukhopadhyay, S.; Mandal, S. K.; Bhaduri, S.; Armstrong, W. H. Manganese clusters with relevance to photosystem II. Chem. Rev. 2004, 104, 3981-4026.

25. Mullins, C. S.; Pecoraro, V. L. Reflections on small molecule manganese models that seek to mimic photosynthetic water oxidation chemistry. Coord. Chem. Rev. 2008, 252, 416-443. 26. Gerey, B.; Gouré, E.; Fortage, J.; Pécaut, J.; Collomb, M.-N. Manganesecalcium/strontium heterometallic compounds and their relevance for the oxygen-evolving center of photosystem II. Coord. Chem. Rev. 2016, 319, 1-24. 
27. Kanady, J. S.; Tsui, E. Y.; Day, M. W.; Agapie, T. A Synthetic Model of the $\mathrm{Mn}_{3} \mathrm{Ca}$ Subsite of the Oxygen-Evolving Complex in Photosystem II. Science 2011, 333, 733-736.

28. Tsui, E. Y.; Kanady, J. S.; Agapie, T. Synthetic Cluster Models of Biological and Heterogeneous Manganese Catalysts for $\mathrm{O}_{2}$ Evolution. Inorg. Chem. 2013, 52, 13833-13848.

29. Kanady, J. S.; Lin, P.-H.; Carsch, K. M.; Nielsen, R. J.; Takase, M. K.; Goddard, W. A.; Agapie, T. Toward Models for the Full Oxygen-Evolving Complex of Photosystem II by Ligand Coordination To Lower the Symmetry of the $\mathrm{Mn}_{3} \mathrm{CaO}_{4}$ Cubane: Demonstration That Electronic Effects Facilitate Binding of a Fifth Metal. J. Am. Chem. Soc. 2014, 136, 14373-14376.

30. Tsui, E. Y.; Agapie, T. Reduction potentials of heterometallic manganese-oxido cubane complexes modulated by redox-inactive metals. Proc. Natl. Acad. Sci. U.S.A. 2013, 110, 1008410088.

31. Tsui, E. Y.; Tran, R.; Yano, J.; Agapie, T. Redox-inactive metals modulate the reduction potential in heterometallic manganese-oxido clusters. Nat. Chem. 2013, 5, 293-299.

32. Krewald, V.; Neese, F.; Pantazis, D. A. On the magnetic and spectroscopic properties of high-valent $\mathrm{Mn}_{3} \mathrm{CaO}_{4}$ cubanes as structural units of natural and artificial water oxidizing catalysts. J. Am. Chem. Soc. 2013, 135, 5726-5739.

33. Krewald, V.; Neese, F.; Pantazis, D. A. Redox potential tuning by redox-inactive cations in nature's water oxidizing catalyst and synthetic analogues. Phys. Chem. Chem. Phys. 2016, 18, 10739-10750.

34. Lin, P.-H.; Takase, M. K.; Agapie, T. Investigations of the Effect of the Non-Manganese Metal in Heterometallic-Oxido Cluster Models of the Oxygen Evolving Complex of Photosystem II: Lanthanides as Substitutes for Calcium. Inorg. Chem. 2015, 54, 59-64.

35. Zhang, C.; Chen, C.; Dong, H.; Shen, J.-R.; Dau, H.; Zhao, J. A synthetic Mn ${ }_{4}$ Ca-cluster mimicking the oxygen-evolving center of photosynthesis. Science 2015, 348, 690-693.

36. Pantazis, D. A.; Ames, W.; Cox, N.; Lubitz, W.; Neese, F. Two interconvertible structures that explain the spectroscopic properties of the oxygen-evolving complex of photosystem II in the $\mathrm{S}_{2}$ state. Angew. Chem., Int. Ed. 2012, 51, 9935-9940.

37. Bovi, D.; Narzi, D.; Guidoni, L. The $\mathrm{S}_{2}$ State of the Oxygen-Evolving Complex of Photosystem II Explored by QM/MM Dynamics: Spin Surfaces and Metastable States Suggest a Reaction Path Towards the $S_{3}$ State. Angew. Chem., Int. Ed. 2013, 52, 11744-11749. 
38. Isobe, H.; Shoji, M.; Yamanaka, S.; Umena, Y.; Kawakami, K.; Kamiya, N.; Shen, J. R.; Yamaguchi, K. Theoretical illumination of water-inserted structures of the $\mathrm{CaMn}_{4} \mathrm{O}_{5}$ cluster in the $\mathrm{S}_{2}$ and $\mathrm{S}_{3}$ states of oxygen-evolving complex of photosystem II: full geometry optimizations by B3LYP hybrid density functional. Dalton Trans. 2012, 41, 13727-13740.

39. Palenik, G. J. Bond Valence Sums in Coordination Chemistry Using Oxidation State Independent $\mathrm{R}_{0}$ Values. A Simple Method for Calculating the Oxidation State of Manganese in Complexes Containing Only Mn-O Bonds. Inorg. Chem. 1997, 36, 4888-4890.

40. Palenik, G. J. Bond Valence Sums in Coordination Chemistry Using Oxidation State Independent $\mathrm{R}_{0}$ Values. Inorg. Chem. 1997, 36, 122-122.

41. Dismukes, G. C.; Siderer, Y. Intermediates of a polynuclear manganese center involved in photosynthetic oxidation of water. Proc. Natl. Acad. Sci. USA 1981, 78, 274-278.

42. Casey, J. L.; Sauer, K. EPR detection of a cryogenically photogenerated intermediate in photosynthetic oxygen evolution. Biochim. Biophys. Acta Bioenerg. 1984, 767, 21-28.

43. Zimmermann, J. L.; Rutherford, A. W. EPR studies of the oxygen-evolving enzyme of Photosystem II. Biochim. Biophys. Acta Bioenerg. 1984, 767, 160-167.

44. Zimmermann, J. L.; Rutherford, A. W. Electron paramagnetic resonance properties of the $\mathrm{S}_{2}$ state of the oxygen-evolving complex of photosystem II. Biochemistry 1986, 25, 4609-4615.

45. de Paula, J. C.; Beck, W. F.; Miller, A.-F.; Wilson, R. B.; Brudvig, G. W. Studies of the manganese site of photosystem II by electron spin resonance spectroscopy. J. Chem. Soc., Faraday Trans. 1987, 83, 3635-3651.

46. Boussac, A.; Girerd, J.-J.; Rutherford, A. W. Conversion of the spin state of the manganese complex in photosystem II induced by near-infrared light. Biochemistry 1996, 35, 6984-6989.

47. Krewald, V.; Retegan, M.; Neese, F.; Lubitz, W.; Pantazis, D. A.; Cox, N. Spin State as a Marker for the Structural Evolution of Nature's Water-Splitting Catalyst. Inorg. Chem. 2016, 55, 488-501.

48. Retegan, M.; Krewald, V.; Mamedov, F.; Neese, F.; Lubitz, W.; Cox, N.; Pantazis, D. A. A five-coordinate $\mathrm{Mn}(\mathrm{IV})$ intermediate in biological water oxidation: spectroscopic signature and a pivot mechanism for water binding. Chem. Sci. 2016, 7, 72-84.

49. Narzi, D.; Bovi, D.; Guidoni, L. Pathway for Mn-cluster oxidation by tyrosine-Z in the $\mathrm{S}_{2}$ state of photosystem II. Proc. Natl. Acad. Sci. U.S.A. 2014, 111, 8723-8728. 
50. Retegan, M.; Pantazis, D. A. Interaction of methanol with the oxygen-evolving complex: atomistic models, channel identification, species dependence, and mechanistic implications. Chem. Sci. 2016, 7, 6463-6476.

51. Capone, M.; Narzi, D.; Bovi, D.; Guidoni, L. Mechanism of Water Delivery to the Active Site of Photosystem II along the $\mathrm{S}_{2}$ to $\mathrm{S}_{3}$ Transition. J. Phys. Chem. Lett. 2016, 7, 592-596.

52. Neese, F. The ORCA program system. WIREs Comput. Mol. Sci. 2012, 2, 73-78.

53. Staroverov, V. N.; Scuseria, G. E.; Tao, J.; Perdew, J. P. Comparative assessment of a new nonempirical density functional: Molecules and hydrogen-bonded complexes. J. Chem. Phys. 2003, 119, 12129-12137.

54. Bühl, M.; Kabrede, H. Geometries of Transition-Metal Complexes from DensityFunctional Theory. J. Chem. Theory Comput. 2006, 2, 1282-1290.

55. Jensen, K. P. Bioinorganic Chemistry Modeled with the TPSSh Density Functional. Inorg. Chem. 2008, 47, 10357-10365.

56. Orio, M.; Pantazis, D. A.; Petrenko, T.; Neese, F. Magnetic and Spectroscopic Properties of Mixed Valence Manganese(III,IV) Dimers: A Systematic Study Using Broken Symmetry Density Functional Theory. Inorg. Chem. 2009, 48, 7251-7260.

57. Pantazis, D. A.; Orio, M.; Petrenko, T.; Zein, S.; Bill, E.; Lubitz, W.; Messinger, J.; Neese, F. A new quantum chemical approach to the magnetic properties of oligonuclear transition-metal complexes: Application to a model for the tetranuclear manganese cluster of Photosystem II. Chem.-Eur. J. 2009, 15, 5108-5123.

58. Pantazis, D. A.; Orio, M.; Petrenko, T.; Zein, S.; Lubitz, W.; Messinger, J.; Neese, F. Structure of the oxygen-evolving complex of photosystem II: information on the $\mathrm{S}_{2}$ state through quantum chemical calculation of its magnetic properties. Phys. Chem. Chem. Phys. 2009, 11, 6788-6798.

59. Pantazis, D. A.; Krewald, V.; Orio, M.; Neese, F. Theoretical magnetochemistry of dinuclear manganese complexes: broken symmetry density functional theory investigation on the influence of bridging motifs on structure and magnetism. Dalton Trans. 2010, 39, 4959-4967.

60. Ames, W.; Pantazis, D. A.; Krewald, V.; Cox, N.; Messinger, J.; Lubitz, W.; Neese, F. Theoretical evaluation of structural models of the $S_{2}$ state in the oxygen evolving complex of photosystem II: protonation states and magnetic interactions. J. Am. Chem. Soc. 2011, 133, 19743-19757. 
61. van Lenthe, E.; Baerends, E. J.; Snijders, J. G. Relativistic total-energy using regular approximations. J. Chem. Phys. 1994, 101, 9783-9792.

62. van Lenthe, E.; Snijders, J. G.; Baerends, E. J. The zero-order regular approximation for relativistic effects: The effect of spin-orbit coupling in closed shell molecules. J. Chem. Phys. 1996, 105, 6505-6516.

63. van Wüllen, C. Molecular density functional calculations in the regular relativistic approximation: Method, application to coinage metal diatomics, hydrides, fluorides and chlorides, and comparison with first-order relativistic calculations J. Chem. Phys. 1998, 109, 392-399.

64. Pantazis, D. A.; Chen, X. Y.; Landis, C. R.; Neese, F. All-electron scalar relativistic basis sets for third-row transition metal atoms. J. Chem. Theory Comput. 2008, 4, 908-919.

65. Pantazis, D. A.; Neese, F. All-electron basis sets for heavy elements. WIREs Comput. Mol. Sci. 2014, 4, 363-374.

66. Weigend, F.; Ahlrichs, R. Balanced basis sets of split valence, triple zeta valence and quadruple zeta valence quality for $\mathrm{H}$ to $\mathrm{Rn}$ : Design and assessment of accuracy. Phys. Chem. Chem. Phys. 2005, 7, 3297-3305.

67. Weigend, F. Accurate Coulomb-fitting basis sets for H to Rn. Phys. Chem. Chem. Phys. 2006, $8,1057-1065$.

68. Neese, F.; Wennmohs, F.; Hansen, A.; Becker, U. Efficient, approximate and parallel Hartree-Fock and hybrid DFT calculations. A 'chain-of-spheres' algorithm for the Hartree-Fock exchange. Chem. Phys. 2009, 356, 98-109.

69. Grimme, S.; Antony, J.; Ehrlich, S.; Krieg, H. A consistent and accurate ab initio parametrization of density functional dispersion correction (DFT-D) for the 94 elements H-Pu. $J$. Chem. Phys. 2010, 132, 154104.

70. Grimme, S.; Ehrlich, S.; Goerigk, L. Effect of the damping function in dispersion corrected density functional theory. J. Comput. Chem. 2011, 32, 1456-1465.

71. Klamt, A.; Schüürman, D. COSMO: a new approach to dielectric screening in solvents with explicit expressions for the screening energy and its gradient. J. Chem. Soc., Perkin Trans. 2 1993, 799-805.

72. Golub, G. H.; Reinsch, C. Singular value decomposition and least squares solutions. Numer. Math. 1970, 14, 403-420. 
73. White, S. R. Density matrix formulation for quantum renormalization groups. Phys. Rev. Lett. 1992, 69, 2863-2866.

74. White, S. R.; Martin, R. L. Ab initio quantum chemistry using the density matrix renormalization group. J. Chem. Phys. 1999, 110, 4127-4130.

75. Chan, G. K.-L.; Sharma, S. The density matrix renormalization group in quantum chemistry. Annu. Rev. Phys. Chem. 2011, 62, 465-481.

76. Marti, K. H.; Reiher, M. New electron correlation theories for transition metal chemistry. Phys. Chem. Chem. Phys. 2011, 13, 6750-6759.

77. Kurashige, Y.; Chan, G. K.-L.; Yanai, T. Entangled quantum electronic wavefunctions of the $\mathrm{Mn}_{4} \mathrm{CaO}_{5}$ cluster in photosystem II. Nat. Chem. 2013, 5, 660-666.

78. Sharma, S.; Sivalingam, K.; Neese, F.; Chan, G. K.-L. Low-energy spectrum of ironsulfur clusters directly from many-particle quantum mechanics. Nat. Chem. 2014, 6, 927-933.

79. Chan, G. K.-L.; Head-Gordon, M. Highly correlated calculations with a polynomial cost algorithm: A study of the density matrix renormalization group. J. Chem. Phys. 2002, 116, 44624476.

80. Chan, G. K.-L. An algorithm for large scale density matrix renormalization group calculations. J. Chem. Phys. 2004, 120, 3172-3178.

81. Ghosh, D.; Hachmann, J.; Yanai, T.; Chan, G. K.-L. Orbital optimization in the density matrix renormalization group, with applications to polyenes and $\beta$-carotene. J. Chem. Phys. 2008, $128,144117$.

82. Sharma, S.; Chan, G. K.-L. Spin-adapted density matrix renormalization group algorithms for quantum chemistry. J. Chem. Phys. 2012, 136, 124121.

83. Pipek, J.; Mezey, P. G. A fast intrinsic localization procedure applicable for ab initio and semiempirical linear combination of atomic orbital wave functions. J. Chem. Phys. 1989, 90, 4916-4926.

84. Fiedler, M. A property of eigenvectors of nonnegative symmetric matrices and its application to graph theory. Czech. Math. J. 1975, 25, 619-633.

85. Fiedler, M. Algebraic connectivity of graphs. Czech. Math. J. 1973, 23, 298-305.

86. Atkins, J. E.; Boman, E. G.; Hendrickson, B. A Spectral Algorithm for Seriation and the Consecutive Ones Problem. SIAM J. Comput. 1998, 28, 297-310. 
87. Barcza, G.; Legeza, Ö.; Marti, K. H.; Reiher, M. Quantum-information analysis of electronic states of different molecular structures. Phys. Rev. A 2011, 83, 012508.

88. Shoji, M.; Isobe, H.; Shen, J. R.; Yamaguchi, K. Geometric and electronic structures of the synthetic $\mathrm{Mn}_{4} \mathrm{CaO}_{4}$ model compound mimicking the photosynthetic oxygen-evolving complex. Phys. Chem. Chem. Phys. 2016, 18, 11330-11340.

89. Shoji, M.; Isobe, H.; Nakajima, T.; Yamaguchi, K. Full geometry optimizations of the $\mathrm{CaMn}_{4} \mathrm{O}_{4}$ model cluster for the oxygen evolving complex of photosystem II. Chem. Phys. Lett. 2015, 640, 23-30.

90. Orio, M.; Pantazis, D. A.; Neese, F. Density Functional Theory. Photosynth. Res. 2009, $102,443-453$.

91. Askerka, M.; Vinyard, D. J.; Wang, J.; Brudvig, G. W.; Batista, V. S. Analysis of the Radiation-Damage-Free X-ray Structure of Photosystem II in Light of EXAFS and QM/MM Data. Biochemistry 2015, 54, 1713-1716.

92. Shoji, M.; Isobe, H.; Yamanaka, S.; Suga, M.; Akita, F.; Shen, J.-R.; Yamaguchi, K. Theoretical studies of the damage-free $\mathrm{S}_{4}$ structure of the $\mathrm{CaMn}_{4} \mathrm{O}_{s}$ cluster in oxygen-evolving complex of photosystem II. Chem. Phys. Lett. 2015, 623, 1-7.

93. Shoji, M.; Isobe, H.; Yamanaka, S.; Suga, M.; Akita, F.; Shen, J.-R.; Yamaguchi, K. On the guiding principles for lucid understanding of the damage-free $\mathrm{S}_{1}$ structure of the $\mathrm{CaMn}_{4} \mathrm{O}_{5}$ cluster in the oxygen evolving complex of photosystem II. Chem. Phys. Lett. 2015, 627, 44-52.

94. Chernev, P.; Zaharieva, I.; Rossini, E.; Galstyan, A.; Dau, H.; Knapp, E.-W. Merging Structural Information from X-ray Crystallography, Quantum Chemistry, and EXAFS Spectra: The Oxygen-Evolving Complex in PSII. J. Phys. Chem. B 2016, 120, 10899-10922.

95. Baker, M. L.; Timco, G. A.; Piligkos, S.; Mathieson, J. S.; Mutka, H.; Tuna, F.; Kozłowski, P.; Antkowiak, M.; Guidi, T.; Gupta, T.; Rath, H.; Woolfson, R. J.; Kamieniarz, G.; Pritchard, R. G.; Weihe, H.; Cronin, L.; Rajaraman, G.; Collison, D.; McInnes, E. J. L.; Winpenny, R. E. P. A classification of spin frustration in molecular magnets from a physical study of large odd-numbered-metal, odd electron rings. Proc. Natl. Acad. Sci. U.S.A. 2012, 109, 19113-19118.

96. Schnack, J. Effects of frustration on magnetic molecules: a survey from Olivier Kahn until today. Dalton Trans. 2010, 39, 4677-4686. 
97. Yamauchi, T.; Mino, H.; Matsukawa, T.; Kawamori, A.; Ono, T.-a. Parallel Polarization Electron Paramagnetic Resonance Studies of the $\mathrm{S}_{\mathrm{l}}$-State Manganese Cluster in the Photosynthetic Oxygen-Evolving System. Biochemistry 1997, 36, 7520-7526.

98. Sancho-Garcia, J. C.; Adamo, C. Double-hybrid density functionals: merging wavefunction and density approaches to get the best of both worlds. Phys. Chem. Chem. Phys. 2013, 15, 14581-14594.

99. Goerigk, L.; Grimme, S. Double-hybrid density functionals. WIREs Comput. Mol. Sci. 2014, 4, 576-600.

100. Grimme, S. Semiempirical hybrid density functional with perturbative second-order correlation. J. Chem. Phys. 2006, 124, 034108.

101. Goerigk, L.; Grimme, S. Efficient and Accurate Double-Hybrid-Meta-GGA Density Functionals-Evaluation with the Extended GMTKN30 Database for General Main Group Thermochemistry, Kinetics, and Noncovalent Interactions. J. Chem. Theory Comput. 2010, 7, 291-309.

102. Karton, A.; Tarnopolsky, A.; Lamère, J.-F.; Schatz, G. C.; Martin, J. M. L. Highly Accurate First-Principles Benchmark Data Sets for the Parametrization and Validation of Density Functional and Other Approximate Methods. Derivation of a Robust, Generally Applicable, Double-Hybrid Functional for Thermochemistry and Thermochemical Kinetics. $J$. Phys. Chem. A 2008, 112, 12868-12886.

103. Miralles, J.; Daudey, J.-P.; Caballol, R. Variational calculation of small energy differences. The singlet-triplet gap in [Cu2Cl6]2-. Chem. Phys. Lett. 1992, 198, 555-562.

104. Miralles, J.; Castell, O.; Caballol, R.; Malrieu, J.-P. Specific CI calculation of energy differences: Transition energies and bond energies. Chem. Phys. 1993, 172, 33-43.

105. Castell, O.; Caballol, R. Ab Initio Configuration Interaction Calculation of the Exchange Coupling Constant in Hydroxo Doubly Bridged Cr(III) Dimers. Inorg. Chem. 1999, 38, 668-673. 106. Cabrero, J.; Ben Amor, N.; de Graaf, C.; Illas, F.; Caballol, R. Ab Initio Study of the Exchange Coupling in Oxalato-Bridged Cu(II) Dinuclear Complexes. J. Phys. Chem. A 2000, 104, 9983-9989.

107. Kärkäs, M. D.; Verho, O.; Johnston, E. V.; Åkermark, B. Artificial Photosynthesis: Molecular Systems for Catalytic Water Oxidation. Chem. Rev. 2014, 114, 11863-12001. 
108. Limburg, B.; Bouwman, E.; Bonnet, S. Molecular water oxidation catalysts based on transition metals and their decomposition pathways. Coord. Chem. Rev. 2012, 256, 1451-1467.

109. Lohmiller, T.; Cox, N.; Su, J.-H.; Messinger, J.; Lubitz, W. The Basic Properties of the Electronic Structure of the Oxygen-evolving Complex of Photosystem II Are Not Perturbed by $\mathrm{Ca}^{2+}$ Removal. J. Biol. Chem. 2012, 287, 24721-24733.

110. Mukherjee, S.; Stull, J. A.; Yano, J.; Stamatatos, T. C.; Pringouri, K.; Stich, T. A.; Abboud, K. A.; Britt, R. D.; Yachandra, V. K.; Christou, G. Synthetic model of the asymmetric $\left[\mathrm{Mn}_{3} \mathrm{CaO}_{4}\right]$ cubane core of the oxygen-evolving complex of photosystem II. Proc. Natl. Acad. Sci. U.S.A. 2012, 109, 2257-2262.

111. Ruettinger, W.; Yagi, M.; Wolf, K.; Bernasek, S.; Dismukes, G. C. O ${ }_{2}$ evolution from the manganese-oxo cubane core $\mathrm{Mn}_{4} \mathrm{O}_{4}{ }^{6+}$ : A molecular mimic of the photosynthetic water oxidation enzyme? J. Am. Chem. Soc. 2000, 122, 10353-10357.

112. Krewald, V.; Pantazis, D. A. Understanding and tuning the properties of redoxaccumulating manganese helicates. Dalton Trans. 2016, 45, 18900-18908.

113. Ho, J.; Ertem, M. Z. Calculating Free Energy Changes in Continuum Solvation Models. J. Phys. Chem. B 2016, 120, 1319-1329.

114. Ho, J. Are thermodynamic cycles necessary for continuum solvent calculation of pKas and reduction potentials? Phys. Chem. Chem. Phys. 2015, 17, 2859-2868.

115. Chan, M. K.; Armstrong, W. H. Tetranuclear manganese-oxo complex with a $2.7 \AA$ manganese-manganese separation and intramolecular water-( $\mu$-oxygen) hydrogen-bonded contacts: $\left[\mathrm{Mn}_{4} \mathrm{O}_{2}(\mathrm{TPHPN})_{2}\left(\mathrm{H}_{2} \mathrm{O}\right)_{2}\left(\mathrm{CF}_{3} \mathrm{SO}_{3}\right)_{2}\right]\left(\mathrm{CF}_{3} \mathrm{SO}_{3}\right)_{3}$. Possible mode for binding of water at the active site of the oxygen-evolving complex in photosystem II. J. Am. Chem. Soc. 1990, 112, 4985-4986.

116. Dexheimer, S. L.; Klein, M. P. Detection of a paramagnetic intermediate in the $S_{1}$ state of the photosynthetic oxygen-evolving complex. J. Am. Chem. Soc. 1992, 114, 2821-2826.

117. Haddy, A.; Lakshmi, K. V.; Brudvig, G. W.; Frank, H. A. Q-Band EPR of the $\mathrm{S}_{2}$ state of photosystem II confirms an $S=5 / 2$ origin of the X-band $g=4.1$ signal. Biophys. J. 2004, 87, 2885-2896.

118. Stoll, S.; Schweiger, A. EasySpin, a comprehensive software package for spectral simulation and analysis in EPR. J. Magn. Reson. 2006, 178, 42-55. 
119. Bencini, A.; Gatteschi, D. EPR of Exchange Coupled Systems. Springer Verlag: Berlin, 1990; p 287.

120. Cox, N.; Ames, W.; Epel, B.; Kulik, L. V.; Rapatskiy, L.; Neese, F.; Messinger, J.; Wieghardt, K.; Lubitz, W. Electronic Structure of a Weakly Antiferromagnetically Coupled $\mathrm{Mn}^{\mathrm{M}} \mathrm{Mn}^{\mathrm{mI}}$ Model Relevant to Manganese Proteins: A Combined EPR, "Mn-ENDOR, and DFT Study. Inorg. Chem. 2011, 50, 8238-8251.

121. Peloquin, J. M.; Campbell, K. A.; Randall, D. W.; Evanchik, M. A.; Pecoraro, V. L.; Armstrong, W. H.; Britt, R. D. "sMn ENDOR of the $\mathrm{S}_{2}$-state multiline EPR signal of photosystem II: Implications on the structure of the tetranuclear Mn cluster. J. Am. Chem. Soc. 2000, 122, 10926-10942.

122. Zheng, M.; Khangulov, S. V.; Dismukes, G. C.; Barynin, V. V. Electronic structure of dimanganese(II,III) and dimanganese(III,IV) complexes and dimanganese catalase enzyme: a general EPR spectral simulation approach. Inorg. Chem. 1994, 33, 382-387.

123. Cox, N.; Rapatskiy, L.; Su, J.-H.; Pantazis, D. A.; Sugiura, M.; Kulik, L.; Dorlet, P.; Rutherford, A. W.; Neese, F.; Boussac, A.; Lubitz, W.; Messinger, J. Effect of $\mathrm{Ca}^{2+} / \mathrm{Sr}^{2+}$ substitution on the electronic structure of the oxygen-evolving complex of photosystem II: a combined multifrequency EPR, sMn-ENDOR, and DFT study of the $\mathrm{S}_{2}$ state. J. Am. Chem. Soc. 2011, 133, 3635-3648.

124. Su, J.-H.; Cox, N.; Ames, W.; Pantazis, D. A.; Rapatskiy, L.; Lohmiller, T.; Kulik, L. V.; Dorlet, P.; Rutherford, A. W.; Neese, F.; Boussac, A.; Lubitz, W.; Messinger, J. The electronic structures of the $\mathrm{S}_{2}$ states of the oxygen evolving complexes of photosystem II in plants and cyanobacteria in the presence and absence of methanol. Biochim. Biophys. Acta Bioenerg. 2011, 1807, 829-840.

125. Kulik, L. V.; Epel, B.; Lubitz, W.; Messinger, J. "sMn pulse ENDOR at $34 \mathrm{GHz}$ of the $\mathrm{S}_{\text {。 }}$ and $\mathrm{S}_{2}$ states of the oxygen-evolving complex in photosystem II. J. Am. Chem. Soc. 2005, 127, 2392-2393.

126. Kulik, L. V.; Epel, B.; Lubitz, W.; Messinger, J. Electronic structure of the $\mathrm{Mn}_{4} \mathrm{O}_{x} \mathrm{Ca}$ cluster in the $\mathrm{S}_{0}$ and $\mathrm{S}_{2}$ states of the oxygen-evolving complex of photosystem II based on pulse sMn-ENDOR and EPR Spectroscopy. J. Am. Chem. Soc. 2007, 129, 13421-13435. 
127. Lohmiller, T.; Ames, W.; Lubitz, W.; Cox, N.; Misra, S. K. EPR Spectroscopy and the Electronic Structure of the Oxygen-Evolving Complex of Photosystem II. Appl. Magn. Reson. 2013, 44, 691-720.

128. Retegan, M.; Cox, N.; Pantazis, D. A.; Neese, F. A First-Principles Approach to the Calculation of the on-Site Zero-Field Splitting in Polynuclear Transition Metal Complexes. Inorg. Chem. 2014, 53, 11785-11793.

129. Campbell, K. A.; Lashley, M. R.; Wyatt, J. K.; Nantz, M. H.; Britt, R. D. Dual-Mode EPR Study of $\mathrm{Mn}(\mathrm{III})$ Salen and the Mn(III) Salen-Catalyzed Epoxidation of cis- $\beta$ Methylstyrene. J. Am. Chem. Soc. 2001, 123, 5710-5719.

130. Campbell, K. A.; Yikilmaz, E.; Grant, C. V.; Gregor, W.; Miller, A.-F.; Britt, R. D. Parallel Polarization EPR Characterization of the Mn(III) Center of Oxidized Manganese Superoxide Dismutase. J. Am. Chem. Soc. 1999, 121, 4714-4715.

131. Horner, O.; Rivière, E.; Blondin, G.; Un, S.; Rutherford, A. W.; Girerd, J.-J.; Boussac, A. SQUID Magnetization Study of the Infrared-Induced Spin Transition in the $\mathrm{S}_{2}$ State of Photosystem II: Spin Value Associated with the $g=4.1$ EPR Signal. J. Am. Chem. Soc. 1998, 120, 7924-7928.

132. Campbell, K. A.; Peloquin, J. M.; Pham, D. P.; Debus, R. J.; Britt, R. D. Parallel Polarization EPR Detection of an $\mathrm{S}_{1}$-State "Multiline" EPR Signal in Photosystem II Particles from Synechocystis sp. PCC 6803. J. Am. Chem. Soc. 1998, 120, 447-448.

133. Pecoraro, V. L.; Baldwin, M. J.; Caudle, M. T.; Hsieh, W.-Y.; Law, N. A. A proposal for water oxidation in photosystem II. Pure Appl. Chem. 1998, 70, 925-929.

134. Vrettos, J. S.; Brudvig, G. W. Water oxidation chemistry of photosystem II. Phil. Trans. R. Soc. B 2002, 357, 1395-1404.

135. Siegbahn, P. E. M.; Crabtree, R. H. Manganese oxyl radical intermediates and O-O bond formation in photosynthetic oxygen evolution and a proposed role for the calcium cofactor in photosystem II. J. Am. Chem. Soc. 1999, 121, 117-127.

136. Siegbahn, P. E. M. A structure consistent mechanism for dioxygen formation in photosystem II. Chem.-Eur. J. 2008, 14, 8290-8302.

137. Siegbahn, P. E. M. Structures and energetics for $\mathrm{O}_{2}$ formation in photosystem II. Acc. Chem. Res. 2009, 42, 1871-1880. 
138. Siegbahn, P. E. M. Water oxidation mechanism in photosystem II, including oxidations, proton release pathways, $\mathrm{O}-\mathrm{O}$ bond formation and $\mathrm{O}_{2}$ release. Biochim. Biophys. Acta Bioenerg. 2013, 1827, 1003-1019.

139. Raucci, U.; Ciofini, I.; Adamo, C.; Rega, N. Unveiling the Reactivity of a Synthetic Mimic of the Oxygen Evolving Complex. J. Phys. Chem. Lett. 2016, 7, 5015-5021.

140. Pérez Navarro, M.; Ames, W. M.; Nilsson, H.; Lohmiller, T.; Pantazis, D. A.; Rapatskiy, L.; Nowaczyk, M. M.; Neese, F.; Boussac, A.; Messinger, J.; Lubitz, W.; Cox, N. Ammonia binding to the oxygen-evolving complex of photosystem II identifies the solvent-exchangeable oxygen bridge ( $\mu$-oxo) of the manganese tetramer. Proc. Natl. Acad. Sci. U.S.A. 2013, 110, 15561-15566.

141. Cox, N.; Messinger, J. Reflections on substrate water and dioxygen formation. Biochim. Biophys. Acta Bioenerg. 2013, 1827, 1020-1030.

142. Lohmiller, T.; Krewald, V.; Pérez Navarro, M.; Retegan, M.; Rapatskiy, L.; Nowaczyk, M. M.; Boussac, A.; Neese, F.; Lubitz, W.; Pantazis, D. A.; Cox, N. Structure, ligands and substrate coordination of the oxygen-evolving complex of photosystem II in the $S_{2}$ state: a combined EPR and DFT study. Phys. Chem. Chem. Phys. 2014, 16, 11877-11892.

143. Cox, N.; Pantazis, D. A.; Neese, F.; Lubitz, W. Artificial photosynthesis: understanding water splitting in nature. Interface Focus 2015, 5, 20150009. 
For Table of Contents Only

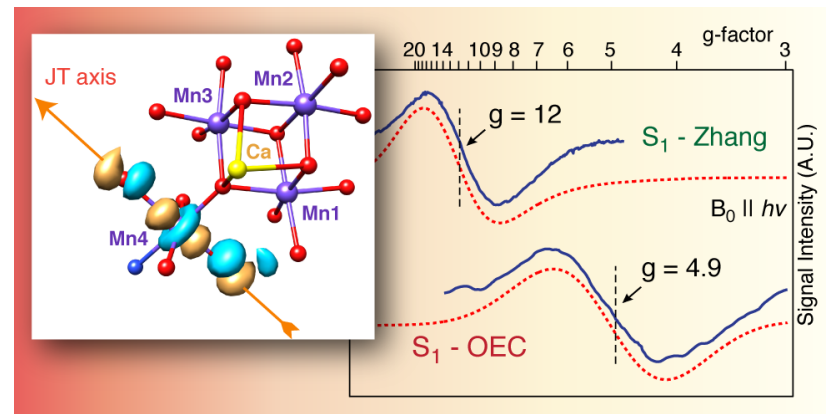

The spectroscopic similarities between the biological oxygen-evolving complex and a synthetic tetramanganese model are shown to have different structural origins. 